\title{
WALKING ASSISTANT - A MOBILE AID FOR THE VISUALLY-IMPAIRED
}

\author{
A Thesis \\ Presented to \\ the Faculty of California Polytechnic State University \\ San Luis Obispo
}

\author{
In Partial Fulfillment \\ of the Requirements for the Degree \\ Master of Engineering in Computer Science
}

by

Adin Miller

June 2014 
(C) 2014

Adin Miller

ALL RIGHTS RESERVED 


\section{COMMITTEE MEMBERSHIP}

TITLE: $\quad$ Walking Assistant - A Mobile Aid for the Visually-Impaired

AUTHOR: $\quad$ Adin Miller

DATE SUBMITTED: June 2014

COMMITTEE CHAIR: Lynne Slivovsky, Ph.D.

Professor of Electrical Engineering

COMMITTEE MEMBER: David Janzen, Ph.D.

Professor of Computer Science

COMMITTEE MEMBER: Franz Kurfess, Ph.D.

Professor of Computer Science 


\begin{abstract}
Walking Assistant - A Mobile Aid for the Visually-Impaired
\end{abstract}

Adin Miller

The most common navigation aid visually-impaired people employ is a white cane, but, recently, technology has given rise to a varied set of sophisticated navigation aids. While these new aids can provide more assistance to a visuallyimpaired person than a white cane, they tend to be expensive due to a small market segment, which in turn can reduce their accessibility. In an effort to produce a technologically-advanced yet accessible navigation aid, an Android application is proposed that detects and notifies users about obstacles within their path through the use of a smartphone's camera. While the smartphone is mounted on a harness worn by the user, the Walking Assistant application operates by capturing images as the user walks, finding features of objects within each frame, and determining how the features have moved from image to image. If it is discovered that an object is moving towards the user, the Walking Assistant will activate the smartphone's vibration mode to alert the user to the object's presence. Additionally, the user can control the Walking Assistant through the use of either touch or voice commands. By conducting real-world tests, it was determined that the Walking Assistant can correctly identify obstacles $42.1 \%$ of the time, while generating false positive obstacle identifications only $15.0 \%$ of the time. The accuracy of the Walking Assistant can be further improved by implementing additional features, such as a fuzzy-decision-based thresholding system or image stabilization.

Keywords: computer vision, smartphone application, optical flow, visuallyimpaired 


\section{TABLE OF CONTENTS}

List of Tables viii

List of Figures $\quad$ ix

I. Introduction 1

II. Background 5

Computer Vision ..................... 5

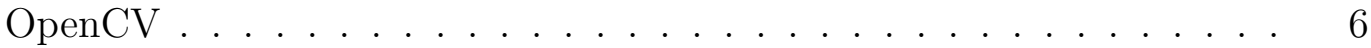

Computer Vision and OpenCV Algorithms . . . . . . . . . . . . 6

Image Pre-Processing . . . . . . . . . . . . . . . . . 6

Feature Point Detection \& Optical Flow . . . . . . . . . . 7

Delaunay Triangulations . . . . . . . . . . . . . . . . 7

Related Works . . . . . . . . . . . . . . . . . . 8

III. Theoretical Framework 11

Shi-Tomasi Corner Detector Method . . . . . . . . . . . . . . . 11

Sub-Pixel Accurate Corner Locator Method . . . . . . . . . . . . . . . 12

Optical Flow . . . . . . . . . . . . . . . . . . . 14

Collision Risk Estimation . . . . . . . . . . . . . . . . . . 15

$\begin{array}{ll}\text { IV. User Experience } & 17\end{array}$

Starting the App . . . . . . . . . . . . . . . . 17

Running the App . . . . . . . . . . . . . . . . . . 19

Voice-Based Operation . . . . . . . . . . . . . . . . . . 19

Touch-Based Operation . . . . . . . . . . . . . . . . . 21

Using the $\mathrm{App} \ldots \ldots \ldots \ldots$. . . . . . . . . . . . . . . . . 21

Closing the App . . . . . . . . . . . . . . . . . . . 23

V. System Architecture 24

Main Function Modules . . . . . . . . . . . . . . . 24

Delaunay Triangulation Modules . . . . . . . . . . . . 26

VI. Implementation Details 30

Starting the App . . . . . . . . . . . . . . 30 
Running the Object Detection and Collision Warning Processes . . . . 32

Stopping the App . . . . . . . . . . . . . . . . . 34

VII. Experimentation and Results 37

Experimentation Test Format _ . . . . . . . . . . . . . 37

Experimental Setup . . . . . . . . . . . . . . . . . 4 40

Results and Analysis . . . . . . . . . . . . . . . . . 40

Pre-Threshold Test Results . . . . . . . . . . . . . . . . . . . . . 40

Single Object Tests . . . . . . . . . . . . . . . . 41

Result Analysis . . . . . . . . . . . . . 42

No Object Tests . . . . . . . . . . . . . . . . . . . 43

Result Analysis . . . . . . . . . . . . . . 44

Shadow Tests . . . . . . . . . . . . . . . 45

Result Analysis . . . . . . . . . . . . 46

Grass Tests . . . . . . . . . . . . . . . . . . 48

Result Analysis . . . . . . . . . . . . . . . . . 48

Pre-Threshold Test Analysis . . . . . . . . . . . . . . . . . . . . 49

Threshold Test Results . . . . . . . . . . . . . . . . . . . . 53

Single Object Tests . . . . . . . . . . . . . . . . 53

Result Analysis . . . . . . . . . . . . . . 54

No Object Tests . . . . . . . . . . . . . . . . . . 54

Result Analysis . . . . . . . . . . . . 55

Shadow Tests . . . . . . . . . . . . . . . 55

Result Analysis . . . . . . . . . . . . . . 56

Grass Tests . . . . . . . . . . . . . . 5 56

Result Analysis . . . . . . . . . . . . . 56 56

Threshold Test Analysis _. . . . . . . . . . . . . . . 57

$\begin{array}{ll}\text { VIII. Conclusion } & 59\end{array}$

Difficulties and Limitations _. . . . . . . . . . . . . 59

Outdoor Environment Difficulties _. . . . . . . . . . . . 60

Hardware Limitations . . . . . . . . . . . . . . . . . . 62 
Optical Flow Limitations . . . . . . . . . . . . . . . . . . 63

Future Work . . . . . . . . . . . . . . . . . . . . 63

Closing Remarks . . . . . . . . . . . . . . . . . . . . . . 64

$\begin{array}{ll}\text { Bibliography } & 66\end{array}$

$\begin{array}{ll}\text { Appendices } & 70\end{array}$

A Walking Assistant Usability Evaluation Outline 71

Evaluation Goals and Objectives . . . . . . . . . . . . . . 71

Methodology . . . . . . . . . . . . . . . . . . 71

Usability Evaluation Plan . . . . . . . . . . . . . . . . . . 71 


\section{LIST OF TABLES}

Table

Page

1 Table of valid commands while choosing an input mode . . . . . . . . 18

2 Table of valid voice commands . . . . . . . . . . . . . . . . . . . . 20

3 Table of valid touch commands . . . . . . . . . . . . . . . . . 22

4 Results of the Pre-Threshold Single Object tests . . . . . . . . . . . . 41

5 Results of the Pre-Threshold No Object tests . . . . . . . . . . . . . 44

6 Results of the Pre-Threshold Shadow tests . . . . . . . . . . . . . . . 46

$7 \quad$ Results of the Pre-Threshold Grass tests . . . . . . . . . . . . . . . . 48

8 Overall results of the Pre-Threshold tests . . . . . . . . . . . . 50

9 Results of the Threshold Single Object tests . . . . . . . . . . . . . . 54

10 Results of the Threshold No Object tests . . . . . . . . . . . . . . 55

11 Results of the Threshold Shadow tests . . . . . . . . . . . . . . . 55

12 Results of the Threshold Grass tests . . . . . . . . . . . . . . . 56

13 Overall results of the Threshold tests . . . . . . . . . . . . . 57 
Figure

\section{LIST OF FIGURES}

1 The UltraCane electronic mobility aid (Ltd 2012) . . . . . . . . . 2

2 The ' $K$ ' Sonar portable electronic mobility aid (of Education 2010) . 2

3 The LIGHBOT guide robot (Hanlon 2013b) . . . . . . . . . . . . . . 2

4 The iGlasses ultrasonice mobility aid (Amazon 2014) . . . . . . . . . 3

5 A Delaunay triangulation in the plane with circumcircles shown (Gjacquenot 2013) . . . . . . . . . . . . . . . . . 8

6 A flowchart displaying the entire system architecture of the Walking Assistant . . . . . . . . . . . . . . . . 25

7 A flowchart displaying the Main Function modules of the Walking Assistant . . . . . . . . . . . . . . . . 27

8 A flowchart displaying the Delaunay Triangulation modules of the Walking Assistant . . . . . . . . . . . . . . . . . . 29

9 A flowchart displaying an overview of the Walking Assistant's entire

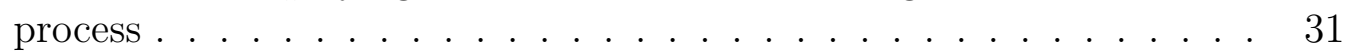

10 A flowchart displaying an overview of the Walking Assistant's object detection process . . . . . . . . . . . . . . . . 35

11 A flowchart displaying an overview of the Walking Assistant's collision warning process . . . . . . . . . . . . . 36

12 Testing environments for each individual test . . . . . . . . . . . . . . 39

13 The Go Pro Harness and smartphone mount used for testing . . . . . 40

14 Feature point detection example for Waypoint tests . . . . . . . . . . 42

15 Feature point detection example for Fire Hydrant tests . . . . . . . . 43

16 Feature point detection example for Bike Locker tests . . . . . . . . . 43

17 Feature point detection example for Cobblestone tests . . . . . . . . . 45

18 Feature point detection example for Shadow Walk-Through tests . . . 47

19 Feature point detection example for Scattered Shadows tests . . . . . 47

20 Feature point detection example for Grass Only tests . . . . . . . . . 49

21 Scatter plot of Pre-Threshold CP, TTC pairs by test type . . . . . . 51

22 Distribution of true positive and false positive collision point values in the Pre-Threshold tests . . . . . . . . . . . . . . . . 52 
23 Distribution of true positive and false positive time-to-collision values in the Pre-Threshold tests . . . . . . . . . . . . . . . . . . 52

24 An example of how a shadow can hide a potential obstacle . . . . . 61 


\section{Introduction}

For visually-impaired people, the common method employed to navigate through an outdoor environment is to use a white cane. Through the use of a white cane, visually-impaired people can detect objects in their path by swinging their cane in an arc and detecting objects based on if their cane hits something within the arc. While the techniques associated with a white cane have gradually been refined over the past couple of decades, they are not entirely without flaws. Specifically, depending on the speed at which one walks and the length of the white cane being used, a visually-impaired person may have only a small window of time to react to the stimulus they receive when their white cane hits an object and avoid the object.

Researchers have tried to improve upon how a visually-impaired person navigates through their environment through the use of technology; some examples of this include technologically-advanced white canes and guide robots. Technologically advanced white canes mimic the functionality of a white cane, but, in the case of the UltraCane (Figure 1), it uses ultrasonic waves to detect obstacles either in front of or above the user (Ltd ).

Other variations on the white cane include the Sonar Traveller Cane, which builds off of a standard white cane by adding sonar technology to detect low hanging objects and determine the distance to an object (McGirr ). Instead of building extra features into the cane itself, the ' $\mathrm{K}$ ' Sonar (Figure 2) can clip onto a white cane and can detect objects up to five meters away. As it is only an attachment, the ' $\mathrm{K}$ ' Sonar can also be used to search for objects around the user, similar to the "way sighted people use a flashlight" (Zabonne ). 


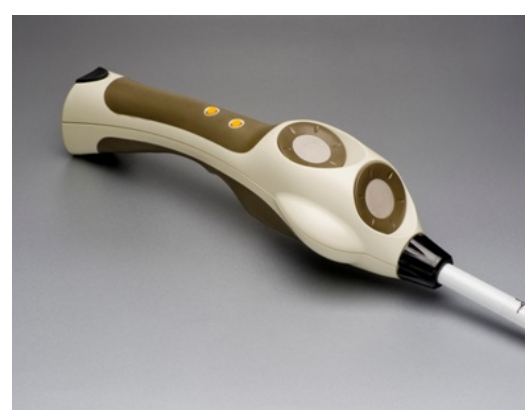

Figure 1: The UltraCane electronic mobility aid (Ltd 2012)

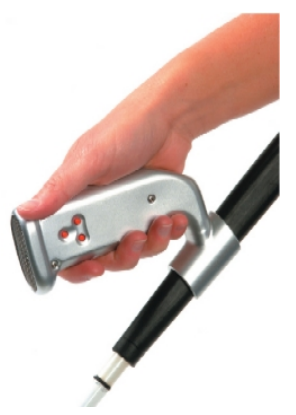

Figure 2: The ' $K$ ' Sonar portable electronic mobility aid (of Education 2010)

In regards to guide robots, the Japan-based company, NSK Ltd. has produced a series of wheeled guide robots. Their most recent iteration, LIGHBOT (Figure 3), guides the visually-impaired like a guide dog would, allowing the user to control the direction they want to go and how fast they want to move (Hanlon 2013a).

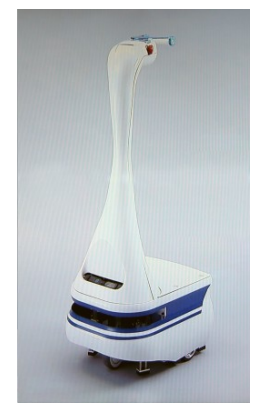

Figure 3: The LIGHBOT guide robot (Hanlon 2013b) 
Another example of assistive technology for visually-impaired users is the iGlasses ultrasonic mobility aid (Figure 4), which are glasses that, like the UltraCane, uses ultrasonic waves and acts as a secondary mobility device, rather than a primary one (AmbuTech 2014).

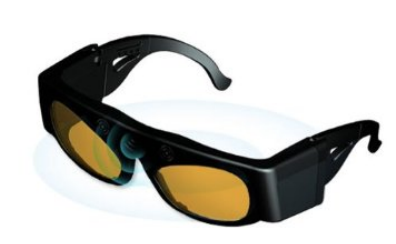

\section{Figure 4: The iGlasses ultrasonice mobility aid (Amazon 2014)}

The main theme throughout all of these examples is that the mentioned equipment must be specially designed and thus, can be quite costly. Additionally, the accessibility of these examples can also be hindered by size and ease of use.

To overcome obstacles presented by the assistive technology listed above, this thesis looks to smartphones and their applications (apps) for several reasons. First, smartphones are ubiquitous; according to an article from the website Business Insider, by the end of 2013, "one out of 5 people in the world own a smartphone" (Heggestuen 2013). Secondly, the average smartphone is designed to fit in a person's pocket, so their size is typically small. Third, smartphone applications can be specially designed for easy use and require less effort to build than designing and constructing, for example, a guide robot. Fourth, smartphones have the ability to recognize speech and can produce noise on cue. Lastly, smartphones and their apps tends to be less expensive than specially designed equipment. Additionally, it is standard for smartphones to come equipped with a camera, which is a necessity when performing computer vision computations. Plus, the processing power of smartphones are increasing with each year, increasing their capability to carry out complex computations.

By creating a smartphone app, this thesis sets out to help visually-impaired 
users better navigate an outdoor environment. Specifically, the object of this thesis is to describe and evaluate the specification, design, and implementation of an Android app that uses the camera on a smartphone to detect and inform visually impaired users about obstacles in their path.

The app, further known as the Walking Assistant, is geared towards expanding upon how a white cane is used, rather than replacing it altogether. The Walking Assistant works by scanning the path the user is traveling through the smartphone's camera, looking for potential obstacles outside of the reach of the user's white cane. If an obstacle is identified and is determined to be moving towards the user, the smartphone will alert the user by vibrating their phone. Therefore, the visually-impaired user won't have to rely solely on their white cane to find obstacles in their path.

This thesis features nine chapters: Introduction, Background, Theoretical Framework, Scenarios of System Use, Overall System Design, Technical Details, Experimentation and Results, and Conclusion.

In Chapter 2, Background, the background of the Walking Assistant is discussed, covering employed computer vision techniques and related research. In Chapter 3, Theoretical Framework, the theoretical framework of the Walking Assistant is described. In Chapter 4, Scenarios of System Use, a step-by-step walkthrough of how the Walking Assistant works from the user's perspective is given. In Chapter 5, Overall System Design, the control flow of the Walking Assistant is described in a moderately technical manner. In Chapter 6, Technical Details, an in-depth technical description of the Walking Assistant is given, describing and explaining the techniques and algorithms used. In Chapter 7, Experimentation and Results, the experiments performed on the Walking Assistant and the results of said experiments are described, followed by an analysis of said results. Lastly, in Chapter 8, Conclusion, the following items are covered: descriptions and analyses of difficulties and limitations associated with the app, potential future work, and an overall conclusion. 


\section{Background}

The Walking Assistant makes use of the Open Source Computer Vision Library, OpenCV, which features "a comprehensive set of both classic and state-of-the-art computer vision and machine learning algorithms," while the Android operating system $^{1}$ is used to provide the interface between the user and the underlying technology behind the app and smartphone (Itseez 2014).

\section{Computer Vision}

In general terms, the field of computer vision involves using computers to analyze and obtain information from images, like humans do every day. As a human, one can easily glean information about one's surrounding environment using our sense of sight, something which comes as first nature. Trying to program a computer to do this is extremely challenging for plethora of reasons, but the field of computer vision is dedicated to solving this problem.

Research in the field of computer vision started as far back as 1951 and one of the earliest accomplishments was the creation of a program that could produce "three-dimensional models of scenes consisting of polyhedral objects" (Eklundh and Christensen 2001). Since then, research has expanded into areas such as information extraction, pattern recognition, and "tracking and real-time analysis of dynamic imagery" (Eklundh and Christensen 2001). Unfortunately, the world has a dynamic and ever-changing nature, thus, all of the major achievements in computer vision have struggled with being robust in dealing with all kinds of

\footnotetext{
${ }^{1}$ The Android operating system was chosen over iOS due to the amount of accessibility to the underlying hardware Android allows, unlike iOS.
} 
real-world data. For example, a computer may be able to detect a user's hand in a room that is brightly lit, but this task becomes much more difficult when the user is in an outdoor environment where the amount of light can change over time or due to the weather conditions.

The main push behind trying to achieve such a goal is that computers can process information and make decisions much faster than humans can, thus, the potential payoff of teaching a computer to gather information and data from images is enormous due to its wide applicability. For example, computer vision could be used to find a person of interest within a crowd or help a driver keep an eye on his surroundings, areas where humans may lose attentiveness or can only do so much.

\section{OpenCV}

The Open Source Computer Vision Library (OpenCV) is an open source library that features implementations of various well-known algorithms for analyzing images and video. OpenCV was originally developed by a team from Intel with the goal of creating "a library of [...] optimized and portable [computer vision] code" that would help "advance [computer] vision research and disseminate [computer] vision knowledge," and since then has been downloaded over 2.5 million times

and features over 2,500 optimized algorithms (Culjak, Abram, Pribanic, Dzapo, and Cifrek 2012). The app presented in this paper, the Walking Assistant, makes use of the library's image pre-processing, feature point detection, and optical flow methods to detect obstacles in the user's path.

\section{Computer Vision and OpenCV Algorithms}

\section{Image Pre-Processing}

Image pre-processing in computer vision involves editing or preparing an image such that the following processing and analysis runs smoothly and produces viable 
results. Specifically, this can include changing the size of the image, converting the image from one color space to another, or "thresholding" an image such that a group of pixels are painted white according to some set threshold and the remaining pixels are painted black. Section VI. discusses the image pre-processing techniques used within the context of the Walking Assistant.

\section{Feature Point Detection \& Optical Flow}

One way to detect objects within an image is to find corners within an image and mark them as feature points if the corner meets a set threshold. An object can then be tracked in successive images through the use of optical flow and the previous image's feature points. Specifically, optical flow determines how an object has moved within an image relative to the camera that captured the image, by attempting to find the same corners that were marked as feature points in the first image. Thus, the movement of an object can be determined based on the movement of feature points from image to image. In its object detection process, the Walking Assistant uses OpenCV's goodFeaturesToTrack function to gather feature points within an image and uses OpenCV's calcOpticalFlowPyrLK function to calculate the optical flow between two images. A deeper explanation of how both functions work can be found in Sections III. and III., respectively.

\section{Delaunay Triangulations}

As a part of modeling the Walking Assistant's collision warning process after those proposed by Shrinivas Pundlik and Gang Luo and Pundlik et al, a twodimensional Delaunay triangulation is used to find the feature point neighbors of each found feature point(Pundlik and Luo 2012; Shrinivas Pundlik and Luo 2013). According to Steven Fortune, a two-dimensional Delaunay triangulation is constructed from a collection of Delaunay triangles, each of which is defined by three feature points that all lie on the edge of an empty circumcircle, as shown in Figure 5 (Fortune 1997). The choice to use a Delaunay triangulation stems from how its resulting graph has applications for nearest neighbor problems and its 
use in representing surface morphology in that it can help "capture the position of linear features that play an important role in a surface" (Oudot ; Ersi ).

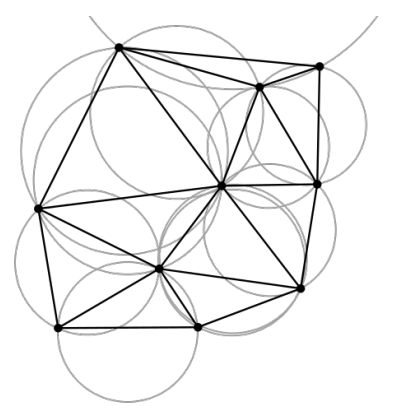

Figure 5: A Delaunay triangulation in the plane with circumcircles shown (Gjacquenot 2013)

\section{Related Works}

In (Song and Huang 2001), Kai-Tai Song and Jui-Hsiang Huang describe a "novel fast optical flow estimation algorithm" and an obstacle avoidance algorithm for a laboratory-developed guide robot for the visually-impaired. The algorithm uses the proposed optical flow algorithm, which "is fast and accurate enough to be applied to real-time obstacle avoidance," and calculates scene depth and TTC to generate a safety distribution histogram, which is used to help the robot determine in which direction to move at any given moment. Despite developing a fast optical flow algorithm, as the robot is custom-designed, its accessibility is more limited than a smartphone application.

Compared to Song and Huang's work, Dejing Ni et al. present a much more robust and more widely applicable walking assistant system for visually-impaired people (Ni, Wang, Ding, Zhang, Song, and Wu 2013). Through the use of a Kinect camera, ultrasonic sensors, GPS navigation, and a vibrotactile belt, the system helps a visually-impaired user navigate through an outdoor environment by altering users about uneven pavement and safe areas in their path - by using a Kinect camera to determine the "safe direction," ultrasonic sensors to determine pavement evenness, and a vibrotactile belt to indicate the safe direction and 
pavement unevenness - and helping the user navigate to their destination - by receiving voice input to indicate destination, using GPS navigation to obtain directions, and using a wireless headset to vocally state navigation directions. Like Song and Huang's guide robot though, Ni et al.'s walking assistant system also has limited accessibility - potentially even more so - and may be too cumbersome due to its setup.

Ying Jie and Song Yanbin describe an obstacle detection algorithm implemented for an electronic travel aid system that also features "road deviation detection" and traffic lights recognition (Jie and Yanbin 2012). The algorithm uses a "self-adaptive threshold determination algorithm" to find obstacles within an image and uses stereo vision, or two cameras, to measure the distance of each object from the user and alert the user if the obstacle is within five meters. Jie and Yanbin's obstacle detection algorithm boasts a $96 \%$ detection rate, but it can't be directly implemented on a smartphone as smartphones typically lack a second camera, which would be needed for distance measurement.

In (Liyanage and Perera 2012), D. Liyanage and M. Perera propose a navigation system for visually-impaired people using much of the same techniques used in this paper and even provides both auditory and tactile feedback through speech synthesis and a microcontroller, respectively. The system uses the Horn and Schunck algorithm, which is a dense version of optical flow ${ }^{2}$, and analyzes each point's time-to-collision value to determine whether or not the user should be notified about an obstacle in their path. Neither of the two differences would prove useful for the Walking Assistant app, since the processing power of smartphones is limited. Therefore, the less computationally-intensive techniques used in a smartphone app, the better.

The object detection processes proposed by Shrinivas Pundlik and Gang Luo in (Pundlik and Luo 2012) and Pundlik et al. in (Shrinivas Pundlik and Luo 2013), which builds off of the former, are closely related to that of the

\footnotetext{
${ }^{2}$ The difference between the dense and sparse versions of optical flow is that, with a dense algorithm, all of the pixels within an image are processed, which is therefore slower, unlike a sparse algorithm where not all of the pixels are processed, making it faster than a dense algorithm.
} 
Walking Assistant system as it is based upon these two processes. Both of the processes use "sparse image motion" either through sparse feature points (Pundlik and Luo 2012) or sparse optical flow (Shrinivas Pundlik and Luo 2013). In either case, the use of sparse image motion is more computationally efficient than dense image motion, and is therefore better suited for use within a smartphone application. The two processes differ in that Pundlik and Luo's work uses a fuzzy decision system to determine thresholds for local scale change and average local motion and k-means clustering to group feature points likely that refer to a single object, while Pundlik et al.'s work uses user-defined, tunable thresholds and groups feature points using a Delaunay triangulation. Additionally, Pundlik et al.'s work adds an external gyroscopic sensor to compensate for camera rotation or bouncing that occurs while the person wearing the camera is walking. 


\section{Theoretical Framework}

In order for the Walking Assistant to meet its proposed goal four different methods are required: the Shi-Tomasi corner detector method, the sub-pixel accurate corner locator method, the Lucas-Kanade method, and the collision risk estimation method used in Pundlik and Luo's and Pundlik et al.'s work.

\section{Shi-Tomasi Corner Detector Method}

In OpenCV, the goodFeatures To Track function employs the Shi-Tomasi corner detector method to find "feature points," or well-defined corners, within an image. This method improves on the Harris corner detector, which calculates a "score" based on the difference between the original search window and the moved search window. The Harris corner detector itself builds off of the Moravec corner detector which finds local maximum differences using the following function:

$$
E_{x, y}=\sum_{u, v} w_{u, v}\left[I_{x+u, y+v}-I_{u, v}\right]^{2}
$$

where $E$ is the difference between the original search window and the moved search window, $x$ is the $\mathrm{x}$-direction displacement, $y$ is the $\mathrm{y}$-direction displacement, $w_{u, v}$ is the search window at position $(u, v), I_{u, v}$ the intensity of the original search window, and $I_{x+u, y+v}$ is the intensity of the moved search window.

The Harris corner detector simplifies this function, allowing it to calculate the aforementioned score for the search window. First, Equation III..1 is expanded using the Taylor series to obtain 


$$
E_{x, y}=\sum_{u, v} w_{u, v}\left[x X+y Y+O\left(x^{2}, y^{2}\right)\right]^{2}
$$

where $X=I \oplus(-1,0,1)$ and $Y=I \oplus(-1,0,1)$. Equation III..2 can then be written as

$$
E(x, y)=A x^{2}+2 C x y+B y^{2}
$$

where $A=X^{2} \oplus w, A=Y^{2} \oplus w$, and $A=(X Y) \oplus w$. Following this, Equation III...3 is transformed into

$$
E(x, y)=(x, y) M(x, y)^{T}
$$

where $M=\left[\begin{array}{ll}A & C \\ C & B\end{array}\right]$.

Lastly, using the eigenvalues of $M$, or $\alpha$ and $\beta$, the following function is used to calculate the search window's score:

$$
R=\operatorname{Det}(M)-k \operatorname{Tr}(M)^{2}
$$

where $\operatorname{Tr}(M)=\alpha+\beta=A+B$ and $\operatorname{Det}(M)=\alpha \beta=A B-C^{2}$. If the resulting score $\mathrm{R}$ exceeds a set threshold, the window is marked as a corner (Team $\mathrm{d}$; Harris and Stephens 1988). Instead of using the above function, the Shi-Tomasi method uses

$$
R=\min (\alpha, \beta)
$$

to calculate a pixel's score, which is known to produce better results that the Harris corner detector (Shi and Tomasi 1994; Team d).

\section{Sub-Pixel Accurate Corner Locator Method}

The feature points produced by goodFeaturesToTrack are then refined using the OpenCV function cornerSubPix by finding accurate, sub-pixel corner locations of 
each feature point. In order to obtain these locations, the position of each corner is found by iteratively calculating the new corner position, $q$, that minimizes $\varepsilon_{i}$.

In using cornerSubPix, it first starts with the following equation:

$$
\varepsilon_{i}=D I_{p_{i}}^{T} \times\left(q-p_{i}\right)
$$

where $D I_{p_{i}}^{T}$ is an image gradient at one of the points $p_{i}$ within the neighborhood, or search window, of $q$. Equation III..7 can then be converted into the following system of equations:

$$
\sum_{i}\left(D I_{p_{i}} \times D I_{p_{i}}^{T}\right)-\sum_{i}\left(D I_{p_{i}} \times D I_{p_{i}}^{T} \times p_{i}\right)
$$

where $\varepsilon_{i}$ is set to zero and the gradients are summed within a neighborhood of $q$. Equation III..8 be further reduced by substituting $G$ for the first gradient term and $b$ for the second gradient term:

$$
q=G^{-1} \times b
$$

. Using this process, cornerSubPix iteratively "sets the center of the neighborhood window" at the newly determined $q$ until "the center stays within a set threshold" (Team a).

Through the arguments passed to cornerSubPix, the following aspects of the algorithm can be specified: half of the side length of $q$ 's neighborhood, half of the size of the "dead region in the middle of the search zone over which the summation in [Equation III..8] is not done," and the termination criteria for when the function should stop iterating (Team a). For cornerSubPix, the Walking Assistant specifies a 5 x 5 half-side-length - or a neighborhood of size 11 x 11 - and a -1 x -1 dead zone - or no dead zone. As for the termination criteria, a maximum of twenty iterations and an accuracy of 0.03 are specified. 


\section{Optical Flow}

While OpenCV features a variety of optical flow implementations, the calcOpticalFlowPyrLK function was used in the Walking Assistant. This function employs the iterative Lucas-Kanade method to find a set of feature points within a second image that correspond to the set of feature points found in a first image using goodFeatures ToTrack.

Specifically, the Lucas-Kanade method is an image alignment algorithm that "[moves], and possibly [deforms] a template to minimize the difference between the template and an image" where the sum of the squared between the template, or the first image, and the second image is minimized using

$$
\sum_{x}\left[I(W(x ; p)-T(x)]^{2}\right.
$$

. In Equation III..10, $W(x ; p)$ refers to the "parameterized set of allowed warps," where $x$ is a pixel in the coordinate frame of template $T$ and, in the case of optical flow, $p=\left(p_{x}, p_{y}\right)^{T}$ is a vector of the $x, y$ coordinate plane parameters. Lastly, $I(W(x ; p))$ is the sub-pixel location of $x$ in $I$ as $W(x ; p)$ takes $x$ and maps it to the sub-pixel location $W(x ; p)$ (Baker and Matthews 2004; Lucas and Kanade 1981).

As a part of OpenCV's calcOpticalFlowPyrLK function, the Lucas-Kanade method uses a $3 \times 3$ sub-region of the first image as the template and attempts to find the "optical flow" vector $p$ that produces the position of the sub-region within the second image (Team c). As calcOpticalFlowPyrLK's documentation notes, this process fails to find large motions, so Gaussian pyramids ${ }^{1}$ are used, which "consists of low-pass filtered, reduced density (i.e., downsampled) images of the preceding level of the pyramid, where the base level is defined as the original image" (Team c; Derpanis 2005).

Additionally, a specific variation of calcOpticalFlowPyrLK is employed, which

\footnotetext{
${ }^{1}$ The pyramid gets its name from the weighting function it uses to generate each level of the pyramid, in that the function closely approximates" the Gaussian smoothing function.
} 
allows the user to specify size of the search window and the maximum number of pyramid levels to be used for optical flow (Team b). For the Walking Assistant, a $7 \times 7$ search window is used to search a maximum of 4 pyramid levels.

\section{Collision Risk Estimation}

The collision risk estimation method used in the Walking Assistant is based off the processes used in the papers of Pundlik and Luo and Pundlik et al., which involves breaking a set of feature points into neighborhoods using a Delaunay triangulation and using collision point (CP) and time-to-collision (TTC) values to determine collision risk (Pundlik and Luo 2012; Shrinivas Pundlik and Luo 2013).

After obtaining a second set of corresponding feature points from calcOpticalFlowPyrLK, a Delaunay triangulation is created and used to determine the neighbors of each feature point in the triangulation. The number of neighbors each feature point has is then reduced by removing any neighbors whose distance from the feature point at root of the neighborhood exceeds a set distance threshold. This reduction is based on the idea that the farther apart two feature points are, the less likely it is they both refer to the same object.

With the "closest" neighbors of each feature point identified, the TTC and $\mathrm{CP}$ values that make up the collision risk estimation are calculated on a local, per-neighborhood basis. TTC is calculated by taking the inverse of the scale change, or local expansion value, of each neighborhood. The local expansion value is calculated using

$$
e_{i}=\frac{\sum_{p_{k}, q_{k} \in D\left(p_{i}, q_{i}\right)}\left(\left\|q_{i}-q_{k}\right\|-\left\|p_{i}-p_{k}\right\|\right)}{\sum_{p_{k}, q_{k} \in D\left(p_{i}, q_{i}\right.}\left\|p_{i}-p_{k}\right\|}
$$

where $p_{i}$ is the $i$ th feature point from the first set of feature points, $q_{i}$ is the corresponding feature point in the second set of feature points, and $D\left(p_{i}, q_{i}\right)$ is the set of immediate feature point neighbors for $\left(p_{i}, q_{i}\right)$ (Shrinivas Pundlik and Luo 2013). 
Rather than check the collision risk of each detected object, only the object, or neighborhood, with the highest collision risk is considered as the user should be alerted if there's at least one object in their path. Additionally, by only analyzing a single high-risk object, less work is required to determine if the user should be notified which can make a difference when these calculations must occur quickly.

The object with the highest collision risk is determined to be the neighborhood with the greatest aggregated CP. If the neighborhood's aggregated CP and local average TTC values are both within a set range, then the neighborhood corresponds to an object that the user should be notified about. 


\section{User Experience}

This section describes how the Walking Assistant operates from the perspective of the user. Specifically, this section explains how the user would start, run, and stop the Walking Assistant.

Before continuing any further, it is important to note that the Walking Assistant isn't designed to replace the use of a white cane, but to act as an extension of it. Thus, it is highly recommended that any and all users use the Walking Assistant in conjunction with a white cane. Additionally, for best results, the user should also use a Go Pro Camera harness with a camera mount to hold their smartphone - with the camera facing outwards - when using the Walking Assistant. The app can still be used if the user holds it at a $45^{\circ}$ to the ground, but the use of a Go Pro Camera harness is suggested. Before carrying out the following instructions, it is assumed that the user is wearing their Go Pro Camera harness with a phone mount and is ready to start navigating outdoors using their white cane primarily.

\section{Starting the App}

Initially, the user will turn their smartphone on, navigate to the Walking Assistant app, and launch it. As the Walking Assistant starts up, the app will vocally state what the set input mode is. The input mode for the phone can be set to one of three settings: voice, touch, and not set. Using the voice input mode, the user can control the app using voice commands, while, for the touch input mode, the 


\begin{tabular}{|l|l|l|l|}
\hline Command & Input & Action & Response \\
\hline \hline $\begin{array}{l}\text { Choose voice } \\
\text { input mode }\end{array}$ & $\begin{array}{l}\text { Single tap, } \\
\text { long tap }\end{array}$ & $\begin{array}{l}\text { Sets input } \\
\text { mode to be } \\
\text { voice-based }\end{array}$ & $\begin{array}{l}\text { "Voice input mode } \\
\text { chosen" }\end{array}$ \\
\hline $\begin{array}{l}\text { Choose } \\
\text { touch-based } \\
\text { input mode }\end{array}$ & $\begin{array}{l}\text { Two taps, } \\
\text { long tap }\end{array}$ & $\begin{array}{l}\text { Sets input } \\
\text { mode to be } \\
\text { touch-based }\end{array}$ & $\begin{array}{l}\text { "Touch input mode } \\
\text { chosen" }\end{array}$ \\
\hline Help & $\begin{array}{l}\text { Three taps, } \\
\text { long tap } \\
\text { valid commands }\end{array}$ & $\begin{array}{l}\text { "Valid commands in- } \\
\text { clude: start, stop, } \\
\text { end, close, cancel, and } \\
\text { help. All are voice and } \\
\text { touch commands, ex- } \\
\text { cept for cancel, which } \\
\text { is only a voice com- } \\
\text { mand." }\end{array}$ \\
\hline Close & $\begin{array}{l}\text { Four taps, } \\
\text { long tap }\end{array}$ & $\begin{array}{l}\text { Closes the appli- } \\
\text { cation }\end{array}$ & "Closing app" \\
\hline
\end{tabular}

Table 1: Table of valid commands while choosing an input mode

app is controlled using a series of taps and long taps ${ }^{1}$, where a series of short taps refer to which command should be executed while a long tap confirms the user's command selection. The input mode can be "not set," in that an input mode has yet to be chosen.

Assuming this is the first time the app has been used by the user, he or she can choose their desired input mode by inputting a single tap, followed by a long tap, to choose the voice input mode or choose the touch input mode by inputting two taps, followed by a long tap. Table 1 provides full descriptions of the commands available while choosing an input mode. If, at some point, the user decides he or she wants to change the input mode, it can be reset with a long tap while using either the voice or touch input mode ${ }^{2}$. If the user accidentally taps the screen too many times, whether they're selecting an input mode or entering in a touch command, the smartphone will detect this, reset the number to detected taps, and state "invalid number of clicks detected" and "resetting number of clicks."

\footnotetext{
${ }^{1}$ A long tap refers to the user holding their finger down on the screen for a second or until the smartphone responds by briefly vibrating.

${ }^{2}$ The input mode can only be reset while the object detection process isn't running.
} 


\section{Running the App}

After choosing an input mode, the user can now freely control the app based on their chosen mode. The following two subsections will discuss how to operate the app using the two input modes, voice-based or touch-based operation.

\section{Voice-Based Operation}

With the voice input mode chosen, the user can now control the app through the use of voice commands, but before a voice command can be given, the Voice Recognition API must first be started. The user can start this API by tapping the screen once and the API will respond with a tone, indicating that it is ready to receive a command from the user. After successfully receiving and recording a command, the API will sound a confirmation tone and will take a moment to determine which command the user has given. If the voice command is recognized as a valid command, the smartphone will give the appropriate vocalized response; otherwise, the smartphone will state that it did not recognize the command and restart the Voice Recognition API.

The "start" voice command will start the object detection process and, once the command is recognized, the Walking Assistant will respond by stating "starting object detection in ten seconds." By delaying the start of the object detection process, it allows the user enough time to mount their smartphone in the Go Pro Camera harness. Once the object detection process has been started, it can be stopped by using either the "stop" or "end" voice commands. If the "end" command is given, both the object detection process and the app itself will be stopped, before which the smartphone will state "stopping object detection and closing app." The "stop" command operates almost exactly the same as the end command, but it will only stop the object detection process, but not the app, and cause the smartphone to state "stopping object detection." If either the "stop" or "end" command is given with the object detection process isn't running, nothing will happen besides the smartphone stating "object detection isn't running." 


\begin{tabular}{|c|c|c|}
\hline Command & Action & Response \\
\hline Start & $\begin{array}{l}\text { Starts or restarts the ob- } \\
\text { ject detection and collision } \\
\text { warning processes after a } \\
\text { ten second delay }\end{array}$ & $\begin{array}{l}\text { "Starting object detection } \\
\text { in ten seconds" }\end{array}$ \\
\hline Stop & $\begin{array}{l}\text { Stops the object detection } \\
\text { and collision warning pro- } \\
\text { cesses only if the object de- } \\
\text { tection process is running }\end{array}$ & "Stopping object detection" \\
\hline End & $\begin{array}{l}\text { Stops the object detection } \\
\text { and collision warning pro- } \\
\text { cesses, and closes the appli- } \\
\text { cation only if the object de- } \\
\text { tection process is running }\end{array}$ & $\begin{array}{l}\text { "Stopping object detection } \\
\text { and closing app" }\end{array}$ \\
\hline Close & Closes the application & "Closing app" \\
\hline Cancel & $\begin{array}{l}\text { Stops the voice recognition } \\
\text { activity }\end{array}$ & $\begin{array}{l}\text { "Cancelling voice recogni- } \\
\text { tion" }\end{array}$ \\
\hline Help & $\begin{array}{l}\text { Lists all of the valid voice } \\
\text { commands }\end{array}$ & $\begin{array}{l}\text { "Valid voice commands in- } \\
\text { clude: start, stop, end, } \\
\text { close, cancel, and help. To } \\
\text { reset input mode, input } \\
\text { long tap." }\end{array}$ \\
\hline $\begin{array}{l}\text { Unknown } \\
\text { command }\end{array}$ & $\begin{array}{l}\text { Restarts voice recognition } \\
\text { activity }\end{array}$ & $\begin{array}{l}\text { "Command not recognized. } \\
\text { Please try again at the } \\
\text { tone." }\end{array}$ \\
\hline
\end{tabular}

Table 2: Table of valid voice commands

Other voice commands include the following: the "cancel" command, which stops the Voice Recognition API and causes the smartphone to state "cancelling voice recognition;" the "close" command, which closes the app and causes the smartphone to state "closing app;" and the "help" command, which causes the smartphone to vocally list all of the previously mentioned voice commands. Table 2 provides full descriptions of the commands available when the voice input mode is chosen. 


\section{Touch-Based Operation}

With the touch input mode chosen, the user can now control the app through the use of touch commands. All touch commands are entered using a series of taps and long taps, but what each combination of taps corresponds to depends on whether or not the object detection process is running. Additionally, the "cancel" command is not available while in touch input mode as it is a command specific to touch input mode. Otherwise, the remaining commands operate the same way and have the same vocal responses as their voice command counterparts.

Assuming that the object detection process currently isn't running, the user

can enter either the "start" or the "close" touch commands. The user can give the "start" command by tapping the screen once and following it with a long tap, while the "close" command is given by tapping the screen twice and following it with a long tap. Assuming now that the object detection process is running, the "stop" and "end" touch commands can be entered. The "stop" command is given by tapping the screen once and following it with a long tap, while the "end" command is given by tapping the screen twice and following it with a long tap. Regardless of whether the object detection process is running or not, the "help" command can be given by tapping the screen three times and following it with a long tap. Table 3 provides full descriptions of the commands available when the touch input mode is chosen.

\section{Using the App}

Now that the user fully understands how to control the app, he or she can now use it to better navigate through an outdoor environment. After starting the object detection process and mounting his or her smartphone on their Go Pro Camera harness, the user can now start walking as they normally do by swinging their white cane in an arc in front of them to feel for any obstacles in their path. The Walking Assistant will operate by detecting potential obstacles in front of the user and determining if any of said obstacles will collide with the user. If such 


\begin{tabular}{|c|c|c|c|}
\hline Command & Input & Action & Response \\
\hline Start & $\begin{array}{l}\text { Single tap, long } \\
\text { tap (valid only } \\
\text { if object detection } \\
\text { process isn't run- } \\
\text { ning) }\end{array}$ & $\begin{array}{l}\text { Starts or restarts } \\
\text { the object detec- } \\
\text { tion and collision } \\
\text { warning processes } \\
\text { after a ten second } \\
\text { delay }\end{array}$ & $\begin{array}{l}\text { "Starting object detection } \\
\text { in ten seconds" }\end{array}$ \\
\hline Close & $\begin{array}{l}\text { Two taps, long } \\
\text { tap (valid only } \\
\text { if object detection } \\
\text { process isn't run- } \\
\text { ning) }\end{array}$ & $\begin{array}{l}\text { Closes the appli- } \\
\text { cation }\end{array}$ & "Closing app" \\
\hline Stop & $\begin{array}{l}\text { Single tap, long } \\
\text { tap (valid only } \\
\text { if object detec- } \\
\text { tion process is } \\
\text { running) }\end{array}$ & $\begin{array}{l}\text { Stops the object } \\
\text { detection and } \\
\text { collision warning } \\
\text { processes only } \\
\text { if the object } \\
\text { detection process } \\
\text { is running }\end{array}$ & "Stopping object detection" \\
\hline End & $\begin{array}{l}\text { Two taps, long } \\
\text { tap (valid only } \\
\text { if object detec- } \\
\text { tion process is } \\
\text { running) }\end{array}$ & $\begin{array}{l}\text { Stops the object } \\
\text { detection and } \\
\text { collision warning } \\
\text { processes, and } \\
\text { closes the appli- } \\
\text { cation only if the } \\
\text { object detection } \\
\text { process is running }\end{array}$ & $\begin{array}{l}\text { "Stopping object detection } \\
\text { and closing app" }\end{array}$ \\
\hline Help & $\begin{array}{l}\text { Three taps, long } \\
\text { tap }\end{array}$ & $\begin{array}{l}\text { Lists all of the } \\
\text { valid voice com- } \\
\text { mands }\end{array}$ & $\begin{array}{l}\text { "Valid voice commands in- } \\
\text { clude: Start: one tap, long } \\
\text { tap while object detection } \\
\text { isn't running; Close: two } \\
\text { taps, long tap while ob- } \\
\text { ject detection isn't running; } \\
\text { Stop: one tap, long tap } \\
\text { while object detection is } \\
\text { running; End: two taps, } \\
\text { long tap while object de- } \\
\text { tection is running; Help: } \\
\text { three taps, long tap any- } \\
\text { time; To reset input mode, } \\
\text { input long tap" }\end{array}$ \\
\hline
\end{tabular}

Table 3: Table of valid touch commands 
an obstacle is found, the smartphone will vibrate, alerting the user that there is an obstacle on the ground in front of them. By giving an early warning about an obstacle in their path, the user will know to move more cautiously by using their white cane to feel for the obstacle, and giving them more time to react when they encounter the detected obstacle.

\section{Closing the App}

Using voice or touch, the user can close the Walking Assistant in one of three ways, either by giving the "end" command, giving the "close" command, or by pressing their smartphone's home button to exit out of the app. 


\section{System Architecture}

This section details how all of the modules of the Walking Assistant app work together and explains the purpose of each module. The entire app and its modules can be broken into two main groups: modules that carry out the main functions of the Walking Assistant and modules that create the Delaunay triangulation required for the collision warning process. For a full overview of the Walking Assistant's system design, refer to Figure 6.

\section{Main Function Modules}

The "Main Function" modules set up the app, interact with the user, and execute the object detection and collision warning processes. The user interaction aspect of the app is handled solely by the WalkingAssistantActivity module, which carries out a variety of tasks. Its first task is to setup the entire app, preparing it to receive and act on user input, by creating and allocating the necessary resources to ensure the entire app runs smoothly. Its second task is to respond to received input from the user. As described in Section IV., user input can in the form of either touch or voice commands and the WalkingAssistantActivity will execute the appropriate action for each command. Its third and last task is to start the object detection and collision warning processes - when the specific command is given -, which involves taking pictures with the smartphone's camera and launching threads for each captured image on which the two aforementioned process will operate on.

To take pictures, WalkingAssistantActivity uses the SnapshotCamera module whose only jobs are to capture an image every second and scale the image 


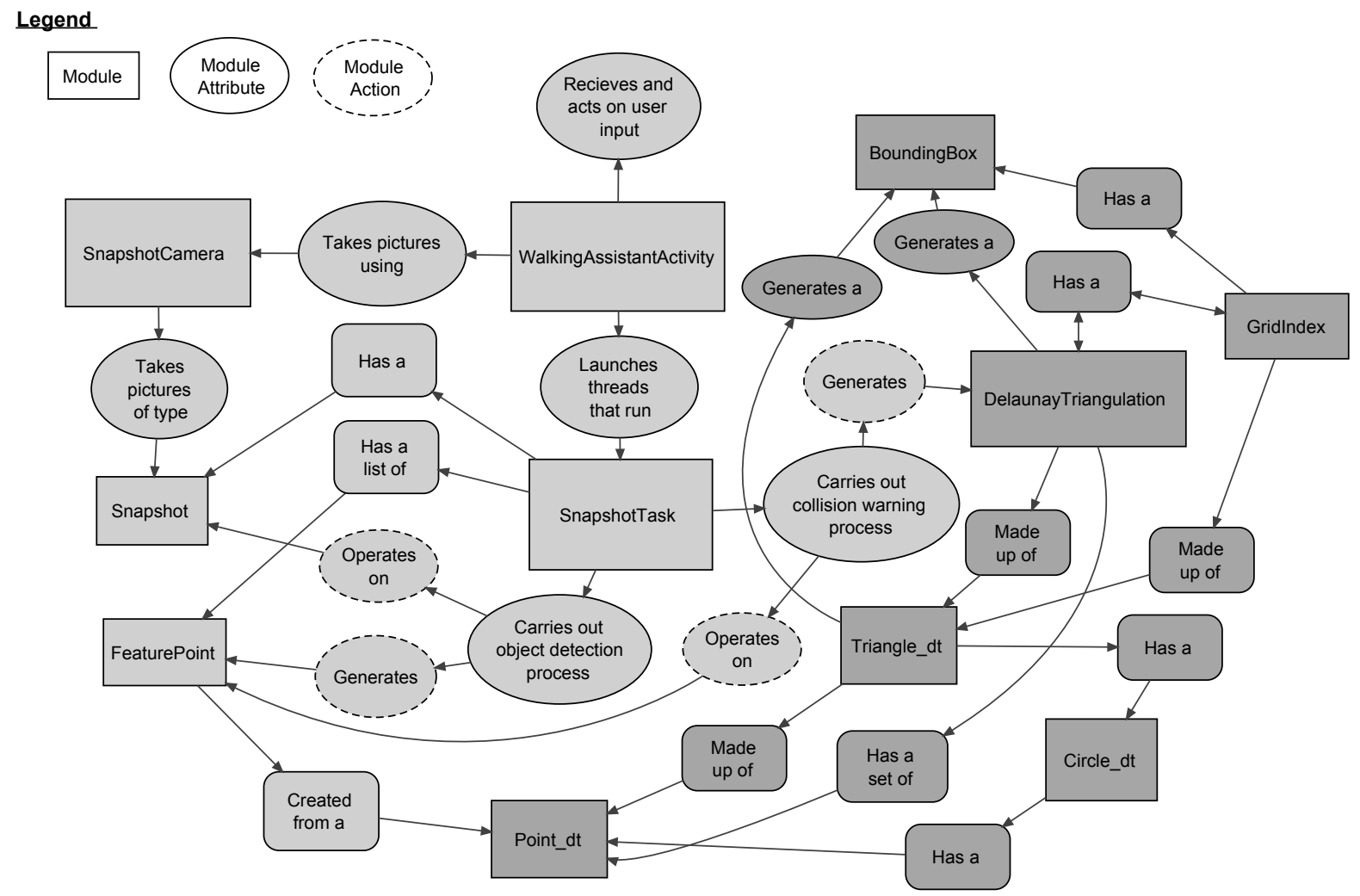

Figure 6: A flowchart displaying the entire system architecture of the Walking Assistant

down to size determined by the dimensions of the smartphone's screen. Each captured image is then passed to a new thread created by WalkingAssistantActivity which carries out the agenda defined by the SnapshotTask module: run the object detection and collision risk processes. One of the module's first tasks is to convert the given image to a Snapshot, which allows SnapshotTask to associate different pieces of information with the image. The SnapshotTask then executes the object detection process by operating on the Snapshot module instance associated with the current thread and the Snapshot instance of another thread. 
During this process, potential obstacles that are found to be in both images are detected and outlined using a collection of generated descriptive points, known as feature points, defined by the FeaturePoint module. An instance of this module holds references to the position of each feature point, as a Java Point object, its corresponding feature point in the second image, a list of feature point neighbors, and collision risk estimation values for the pair of feature points.

Once finished, the object detection process produces a collection of FeaturePoint instances, which SnapshotTask then passes to the collision warning process as input. The collision warning process will break up the collection into groups by creating and using a Delaunay triangulation ${ }^{1}$ to determine if an instance of a FeaturePoint and its FeaturePoint neighbors refer to an object. For each FeaturePoint group, a local collision risk estimation is calculated as to whether or not the object the group refers to will collide with the user within the near future. If a large collision risk estimation is detected, the collision warning process will warn the user about the object's presence by vibrating their smartphone.

A flowchart for the Main Function modules can be seen in Figure 7.

\section{Delaunay Triangulation Modules}

When the SnapshotTask module creates a Delaunay triangulation, it does so by using the Delaunay triangulation module set created by the members of the Java Delaunay Triangulation project(?? ), which includes the following modules: DelaunayTriangulation, BoundingBox, GridIndex, Triangle_dt, Circle_dt, and Point_dt. Of the set, DelaunayTriangulation acts as the main module and the remaining modules are all used to create and support a Delaunay triangulation.

The creation process is started when the SnapshotTask module passes a collection of FeaturePoint instances from the object detection process to the collision warning process, which are then passed to the DelaunayTriangulation module.

\footnotetext{
${ }^{1}$ An in-depth description of a Delaunay triangulation and how they are created can be found in the Section II..
} 


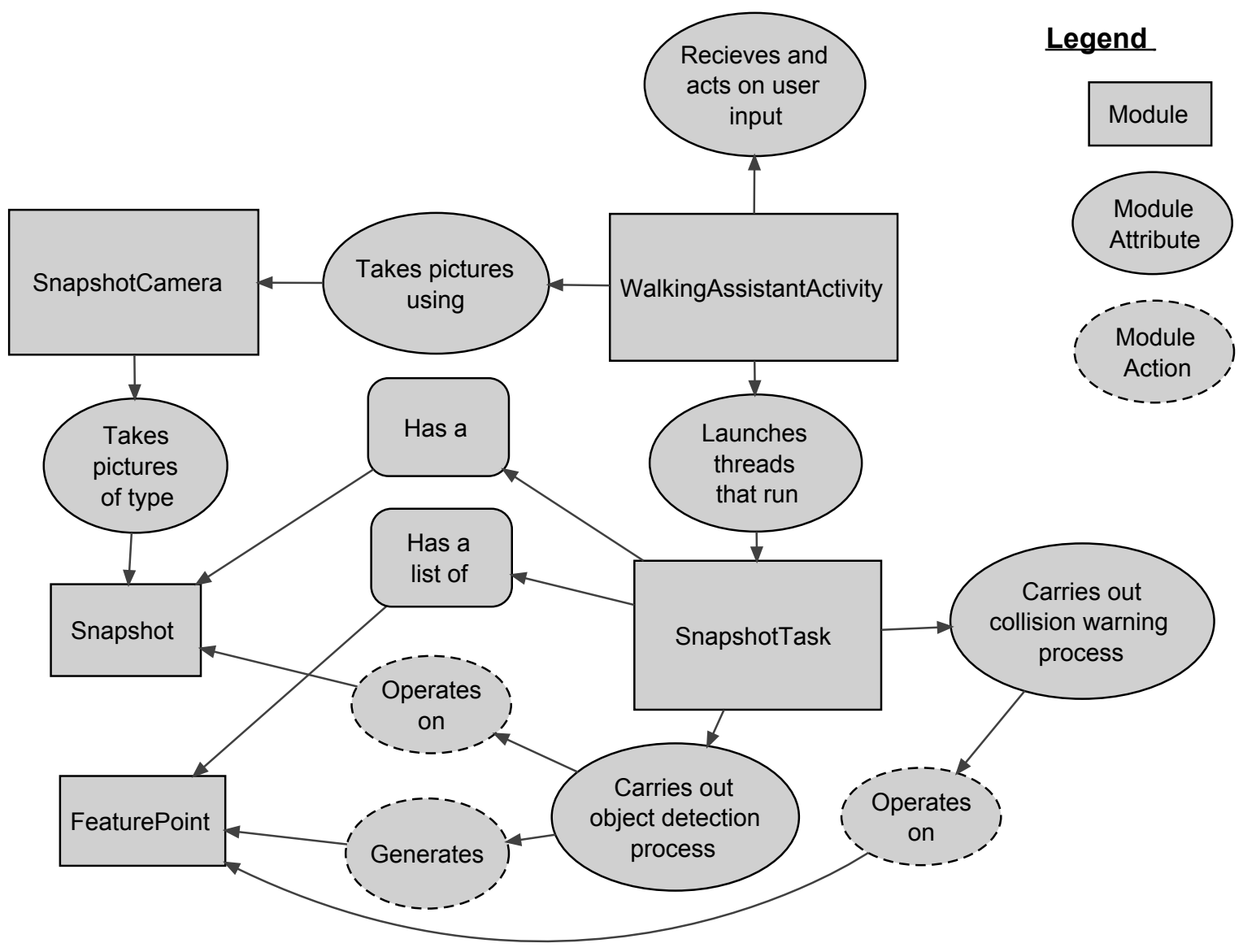

Figure 7: A flowchart displaying the Main Function modules of the Walking Assistant

From these instances, the position of each "corresponding" feature point, in Point form, is converted to an instance of the Point_dt module. This module behaves similarly to that of the Java Point class, but a Point_dt instance can refer to a point in either two-dimensional or three-dimensional space, and the module features extra methods required to turn the Point_dt instances into a Delaunay triangulation.

The newly-converted Point_dt instances are used to create a series of Triangle_dt instances, where each instance is defined by three Point_dt instances and 
a cirumcircle - a Circle_dt instance defined by a Point_dt center and a radius of type double - whose edge all three points lie on. The grid of triangles created by the collection of Triangle_dt instances is what makes up a Delaunay triangulation. As for the GridIndex module, its author, Aviad Segev, gives the following description:

Grid Index is a simple spatial index for fast pointtriangle location. The idea is to divide a predefined geographic extent into equal sized cell matrix (tiles). Every cell will be associated with a triangle which lies inside. [Therefore], one can easily locate a triangle in close proximity of the required point by searching from the point's cell triangle ${ }^{2}$.

The GridIndex module also contains an instance of the BoundingBox module, which defines a bounding box for the entire geographic space covered by a specific GridIndex instance. This bounding box is, in turn, defined by its lower left and upper right corners, which are of type double. The DelaunayTriangulation can also generate a bounding box, but instead of using an instance of the BoundingBox module, it uses two Point_dt instances to define the lower left and upper right corners of the box.

The result of all of these modules working together is a Delaunay triangulation, which the collision warning process can then use to determine which neighbors a feature point has and calculate the collision risk estimation using each feature point and its neighbors.

A flowchart for the Delaunay Triangulation modules can be seen in Figure 8.

\footnotetext{
${ }^{2}$ This quote is taken directly the creator's comments within the source code.
} 


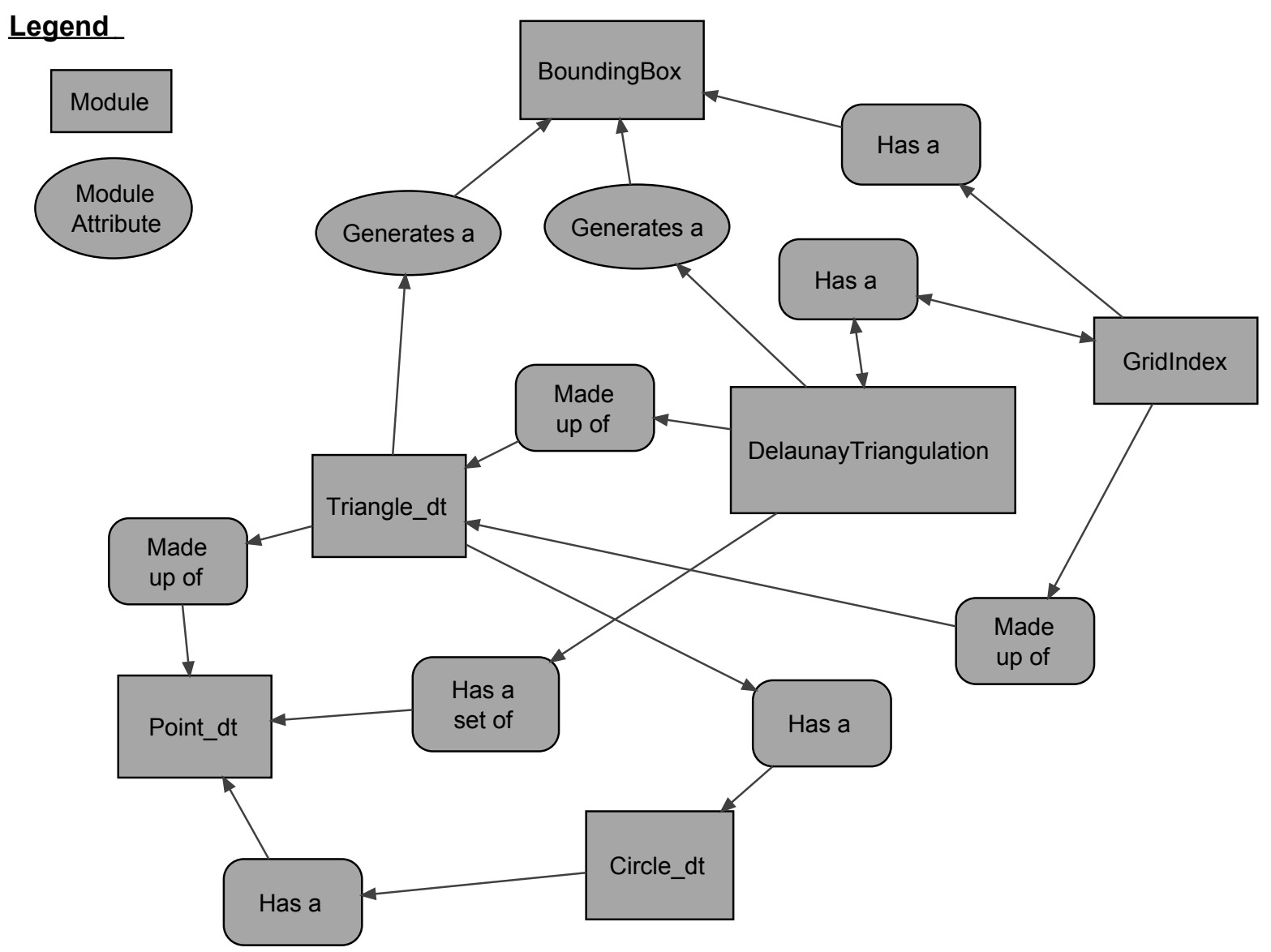

Figure 8: A flowchart displaying the Delaunay Triangulation modules of the Walking Assistant 


\section{Implementation Details}

This section is broken into three parts: starting the Walking Assistant, running object detection and collision warning process, and stopping the app. A full overview of the process flow of the Walking Assistant app can be seen in Figure 9 .

\section{Starting the App}

When the user launches the Walking Assistant, the app will carry out the necessary setup to prepare for user input and to start the object detection and collision warning processes. Once it has finished setting up, the app will await input from the user. Initially, input from the user will come in the form of "taps" (i.e. tapping the screen) and "long taps" (i.e. tapping the screen and holding down), which are used to choose the preferred input mode, either voice or touch. If the user would prefer to use voice commands, the user must tap the screen once and then perform a long tap to confirm their choice to use the voice input mode. Otherwise, if the user chooses to use the touch input mode, the user must tap the screen twice, as opposed to once and follow it with a long tap. In either scenario, the app will vocally state which input mode has been chosen. At this point, the user can start issuing commands or, if they desire, change the chosen input mode. All touch-based commands use a combination of taps and long taps and a full list of touch-based commands are listed in Table 3. If an invalid input combination is entered, the app will reset the tap count and verbally confirm the action.

As for voice commands, Android's SpeechRecognizer activity will listen for 


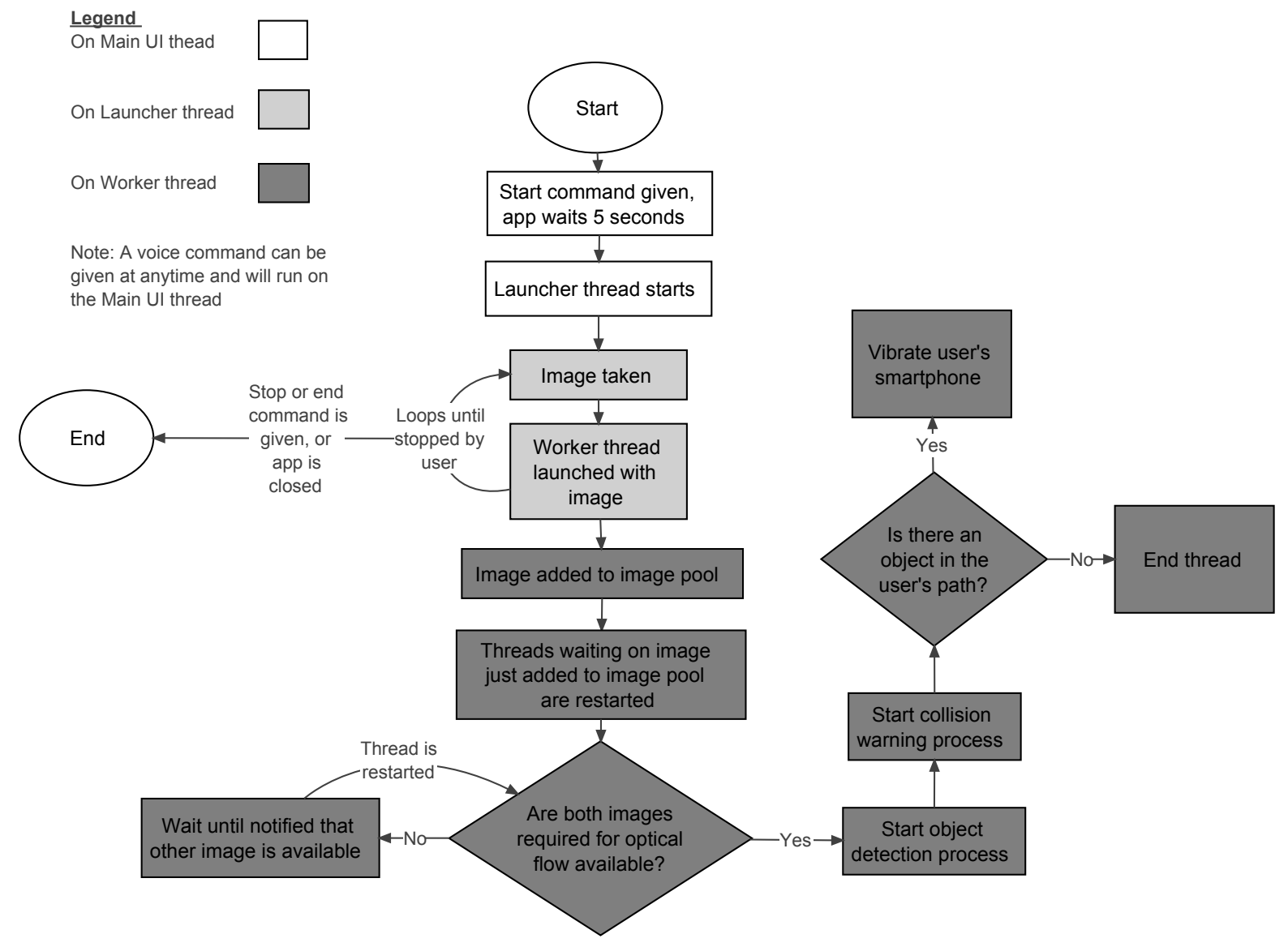

Figure 9: A flowchart displaying an overview of the Walking Assistant's entire process

the command and make a guess as to what the user said. The results of the SpeechRecognizer will be compared against a list of valid commands, as listed in Table 2. If a match is found, the app will carry out the voice command's action and verbally state the command's response through the use of the SpeechToText class. If a match isn't found, the app will carry out the action and verbally say the response for the "unknown command" voice command. To start the app's object detection and collision warning processes, the user must give the "start" 
command, which will first verbally state "starting object detection in ten seconds" before waiting ten seconds ${ }^{1}$ before starting the object detection process.

\section{Running the Object Detection and Collision Warning Processes}

Once the ten seconds are up, the app will create a thread pool and start a new thread, further known as the Launcher thread, to keep the following intensive work off of the main UI thread. On the Launcher thread, every second, a picture is taken and a new thread, further known as a Worker thread, from the thread pool is launched to carry out the object detection and collision warning processes using the recently-taken picture.

Upon being launched, a Worker thread will add their image to the pool of images $^{2}$ available for optical flow calculations and will either pause itself or continue on. Whether the thread pauses itself or not depends on if the chronologicallynext image ${ }^{3}$, which is required to calculate optical flow, is present within the image pool. If the image can't be found in the image pool, the Worker thread will pause itself, through the use of the Object class' wait method, and wait for another Worker thread to notify it, through the use of the Object class' notify method, indicating that the image the former thread requires is now available. Otherwise, the Worker thread will start the object detection process using its own image and the chronologically-next image as input. A flowchart of the object detection process can be seen in Figure 10.

The first step of the four-step object detection process consists of image pre-processing, such as grayscale conversion and cropping. The two images are first converted from the red-green-blue (RGB) color space to grayscale and then cropped down to a smaller size. The two images are cropped horizontally, since

\footnotetext{
${ }^{1}$ The app pauses ten seconds before starting the object detection and collision warning processes to allow the user enough time to place their phone in their Go Pro Camera harness in preparation for using the app.

${ }^{2}$ Since this image pool will be accessed by several different threads, synchronization is employed to avoid memory consistency errors and thread interference(Corporation 2014).

${ }^{3}$ For example, if a picture is taken at time $t=1$, the picture taken at time $t=2$ is required to calculate optical flow.
} 
the app should only detect objects directly in front of the users rather than objects off to the side of the user. Additionally, cropping the two images reduces the amount of work required to analyze each image, since less pixels must be analyzed.

For the second step, a set of feature points are found within the first image using the OpenCV function goodFeaturesToTrack. The locations of said feature points are then refined in order to obtain accurate, sub-pixel positions for the points using OpenCV's cornerSubPix function.

The third step involves calculating the optical flow of the two pictures, by determining how the feature points obtained from the first image have moved in the second image through the use of OpenCV's calcOpticalFlowPyrLK function. For the set of feature points passed to calcOpticalFlowPyrLK, a corresponding set of feature points are found within the second image and is passed back. Status and error values are also returned, as calcOpticalFlowPyrLK can only estimate the positions of the corresponding feature points or it may not find corresponding points at all. The status and error values indicate, respectively, whether a corresponding feature point has been found or not, and the value of the error - the calculated minimum difference between the feature point's subregion in the first image and the corresponding region in the second image - of the corresponding point.

In the fourth step and final step of the object detection process, the amount of feature point pairs are reduced by keeping pairs that were found to have a corresponding point in the second image and have an error value less than that of a set error threshold. The remaining pairs of feature points are then passed to the collision detection process to determine if the user should be notified about any of the detected objects, as defined by the feature point pairs, found within the two images. A flowchart of the collision warning process can be seen in Figure 11.

After the object detection process has produced a set of feature point pairs, the collision warning process breaks the pairs up into neighborhoods based on a Delaunay triangulation created from feature points from the second image. For 
each neighborhood, its CPs values are aggregated and the neighborhood with the highest aggregated $\mathrm{CP}$ value is considered to be the object with the highest collision risk. After calculating the local average TTC of the neighborhood, if both of the aforementioned values fall within a predefined range, the Walking Assistant has identified an object in the user's path and notifies them by vibrating their smartphone.

\section{Stopping the App}

The object detection and collision risk processes will run endlessly until the user stops the app or the app is paused and destroyed by the user's smartphone. The user can stop the two processes at anytime by giving either the "stop" or "end" command through the chosen input mode. Both of these commands stop the two processes and the thread pool, and give a vocal confirmation corresponding to the recognized command, but the end command will also close the app, unlike the stop command. 
Legend

FP - Feature point

FP-f - 1st set of feature points

FP-s - 2nd set of feature points

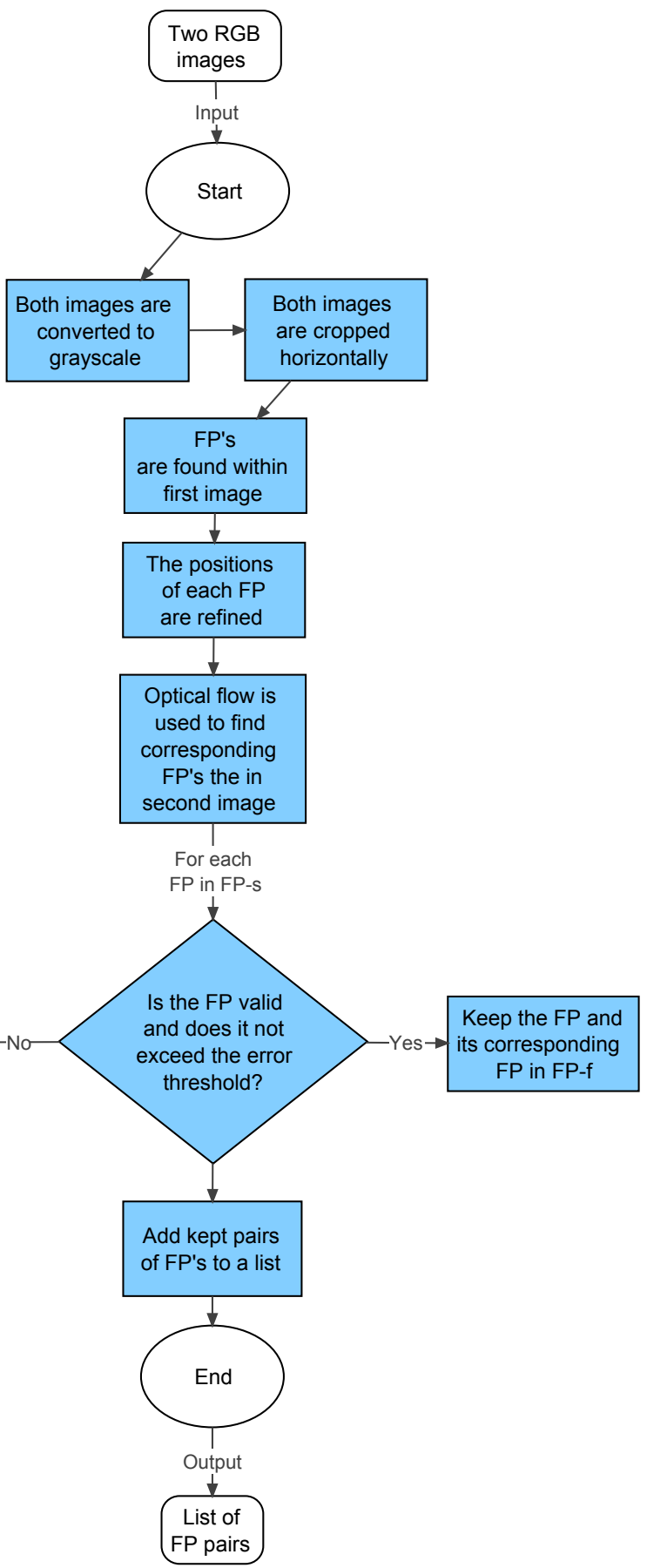

Figure 10: A flowchart displaying an overview of the Walking Assistant's object detection process 


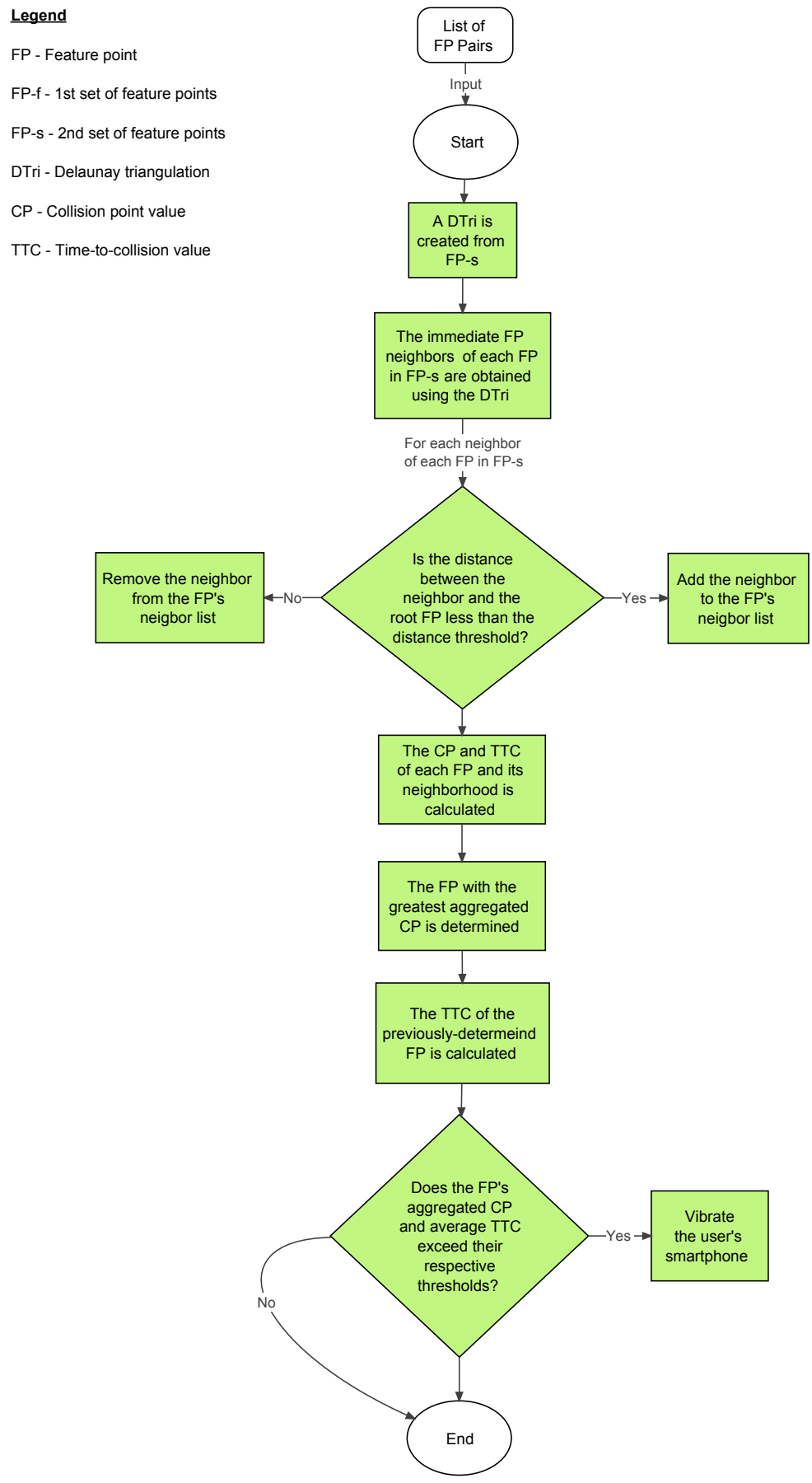

Figure 11: A flowchart displaying an overview of the Walking Assistant's collision warning process 


\section{Experimentation and Results}

The following sections describe the test format and setup of the experimentation carried out using the Walking Assistant. Following this, the results of the tests are then analyzed.

\section{Experimentation Test Format}

In order to test the Walking Assistant, two sets of tests were conducted. The first set of tests is used to determine the thresholds that identify valid CP and TTC values which correspond to a valid detection of an object. The second test set involves testing the newly-set $\mathrm{CP}$ and TTC values.

Each set of tests consists of four different types of tests, which attempt to cover the different situations the average user might encounter while using the Walking Assistant.

The first test type, known as the Single Object tests, deals with detecting a single obstacle in the user's path and is broken up into three individual tests. The first test places a fire hydrant in the user's path (Fire Hydrant), the second uses a map directory sign (Waypoint), while the third uses a row of closely-grouped bicycle lockers (Bike Lockers).

The second test type, known as the Surface Type tests, deals with the different ground surface types a user may walk on and doesn't feature any obstacles; this type is also broken up into three individual tests. The first test occurs on a cobblestone path (Cobblestone), the second occurs on a typical sidewalk (Sidewalk), while the third occurs on an asphalt road (Asphalt). 
The third test type, known as the Shadow tests, deals with the presence of shadows in the user's path and, like the previous test type, it is broken up into three individual tests and doesn't feature any obstacles. The first test deals with if the user is running the Walking Assistant while the user is entirely within a shadow (Full Shadow). The second test involves walking through a single, relatively solid shadow (Shadow Walk-Through), while the third involves walking through scattered shadows (Scattered Shadows).

The fourth and last test type, known as the Grass tests, deals with running the Walking Assistant while the user is walking on grass and is made up of only two tests. The first test involves detecting a fire hydrant on the grass (Grass Obstacle), while the second test doesn't involve detecting an obstacle (Grass Only).

Each of the areas used for testing across the four test types can be seen in Figure 12.

For each pair of images taken during each test, the object detection and collision warning processes produces one of four possible outcomes, which include: a true positive, when an object within the smartphone's camera's view is correctly identified as an obstacle and the user is notified; a false positive, when the user is notified and an obstacle isn't within view or anything other than an obstacle is incorrectly identified as one; a true negative, when the user isn't notified and an obstacle isn't within view; and a false negative, when the user isn't notified and an obstacle is within view. Out of the four outcomes, the true positive, false positive, false negative outcomes produce the most insightful information, so the true negative rates will not be discussed as deeply as the other outcomes.

All of the tests were carried out on sunny days between the hours of 12 p.m. and 5 p.m., so as to avoid sudden changes in lighting caused by clouds and to minimize the length of the shadow cast by the tester due to the angle of the sun. 


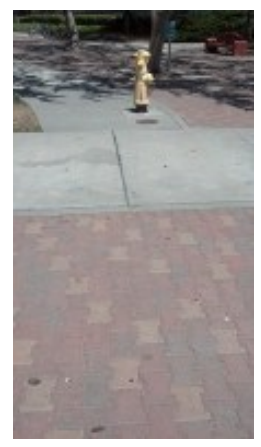

(a) Single Object Test \#1

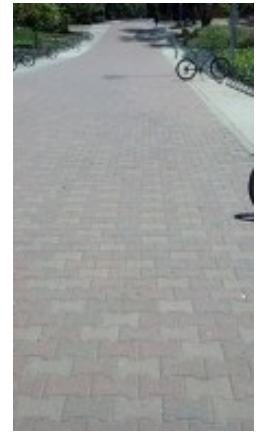

(d) No Object Test \#1

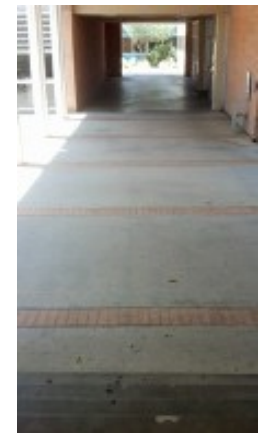

(g) Shadow Test \#1

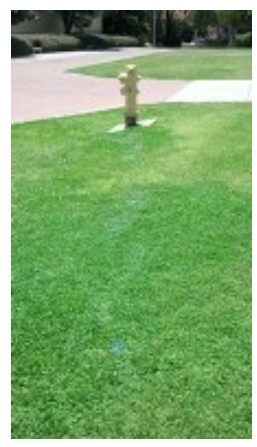

(j) Grass Test \#1

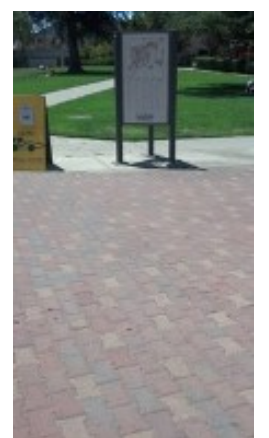

(b) Single Object Test \#2

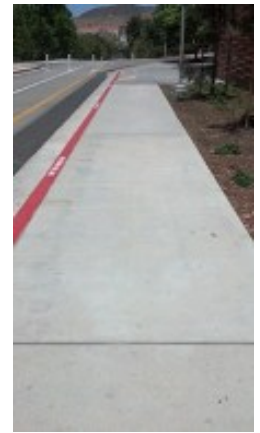

(e) No Object Test \#2

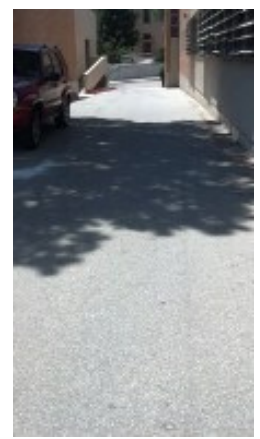

(h) Shadow Test \#2

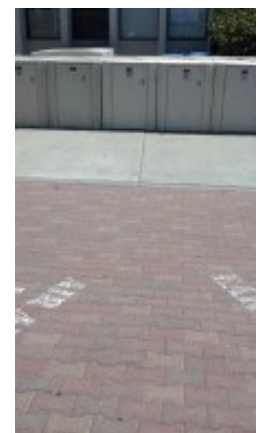

(c) Single Object Test \#3

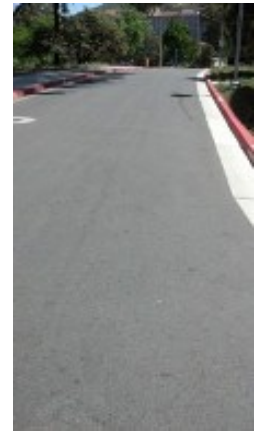

(f) No Object Test \#3

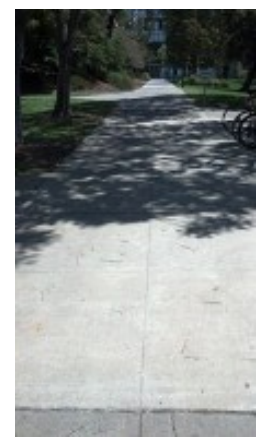

(i) Shadow Test \#3

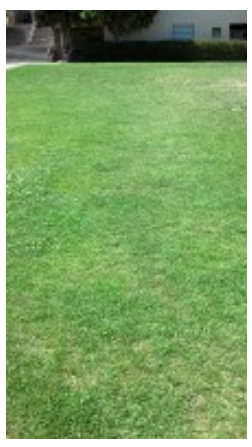

(k) Grass Test \#2

Figure 12: Testing environments for each individual test 


\section{Experimental Setup}

Each test was conducted using the Walking Assistant app on a Motorola Droid 4 smartphone, running on Android's Jelly Bean version 4.1.2 operating system. The smartphone itself was mounted on a modified smartphone mount attached to a Go Pro Chest Harness ${ }^{1}$ worn by the tester, as shown in Figure 13. Additionally, the mount allows the smartphone to be held at an angle, so, during the tests, it was set to its maximum allowable angle to ensure that the smartphone is pointed towards the ground.

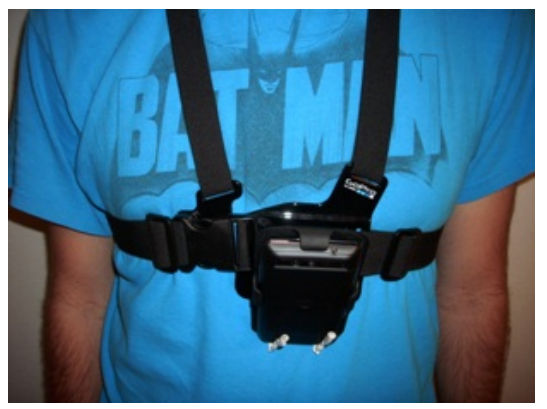

Figure 13: The Go Pro Harness and smartphone mount used for testing

\section{Results and Analysis}

\section{Pre-Threshold Test Results}

As CP and TTC thresholds have yet to be determined, for all of the pre-threshold tests, all positive CP and TTC values are seen as valid values. Thus, the user will be notified about the presence of an object in their path if any of the feature points and its neighbors have positive CP and TTC values.

\footnotetext{
${ }^{1}$ The smartphone mount had to modified since, in its current orientation, it lacks a clip to keep the smartphone it holds from sliding out of the mount. This was accomplished using a clothes hanger, which was bent to wrap around the mount.
} 


\begin{tabular}{|l|l|l|l|l|l|}
\hline Test \# & $\begin{array}{l}\text { Correct } \\
\text { ID }\end{array}$ & $\begin{array}{l}\text { Incorrect } \\
\text { ID }\end{array}$ & $\begin{array}{l}\text { True } \\
\text { Positive }\end{array}$ & $\begin{array}{l}\text { False } \\
\text { Positive }\end{array}$ & $\begin{array}{l}\text { False } \\
\text { Negative }\end{array}$ \\
\hline \hline 1 & $54.5 \%$ & $45.5 \%$ & $40.0 \%$ & $33.3 \%$ & $26.7 \%$ \\
\hline 2 & $100.0 \%$ & $0.0 \%$ & $60.0 \%$ & $0.0 \%$ & $33.3 \%$ \\
\hline 3 & $100.0 \%$ & $0.0 \%$ & $73.3 \%$ & $0.0 \%$ & $13.3 \%$ \\
\hline
\end{tabular}

Table 4: Results of the Pre-Threshold Single Object tests

\section{Single Object Tests}

Each Single Object test features a different obstacle, but initially, the obstacle isn't within the view of the smartphone's camera. Thus, a percentage of the images taken result in "true negatives user notifications", where a user notification doesn't occur because an actual obstacle isn't detected. This is unlike a false positive user notification where a user notification occurs even though an obstacle isn't within the camera's view.

For the Fire Hydrant tests, $54.5 \%$ of the user notifications - when the Walking Assistant notifies the user about an obstacle in their path - were caused by true positive outcomes $^{2}$, but the fire hydrant went undetected $26.7 \%$ of the time. The false positive rate for the tests was $33.3 \%$, because the ground in front of the user was falsely identified as an obstacle. For the Waypoint tests, $100 \%$ of the user notifications resulted from true positive outcomes. In comparison to the Fire Hydrant tests, the Waypoint tests had a higher amount of true positive outcomes - $60 \%$ compared to $0 \%$ - but a higher amount of false negative outcomes $-33.3 \%$ compared to $26.7 \%$. The Bike Locker tests had the best true positive and false negative rates, which were $73.3 \%$ and $13.3 \%$, respectively. The results for all of the Pre-Threshold Single Object tests can be seen in Table 4, where Test \#1 refers to the Fire Hydrant tests, Test \#2 refers to the Waypoint tests, and Test \#3 refers to the Bike Locker tests.

\footnotetext{
${ }^{2}$ In the context of Table 4, this is known as a "Correct ID". An "Incorrect ID" is a user notification that is caused by a false positive outcome.
} 


\section{Result Analysis}

The Waypoint tests had a higher true positive outcome rate in comparison to the Fire Hydrant tests because optical flow typically generated a higher number of feature points clustered around the obstacle in the second test as opposed to the first, which can be seen ${ }^{3}$ in Figures 14 and 15, respectively. Thus, in the Waypoint tests, a feature point's neighborhood tended to be larger thereby likely generating larger CP and TTC values for a feature point and its neighborhood, which the collision warning process would be more likely to pick up on.

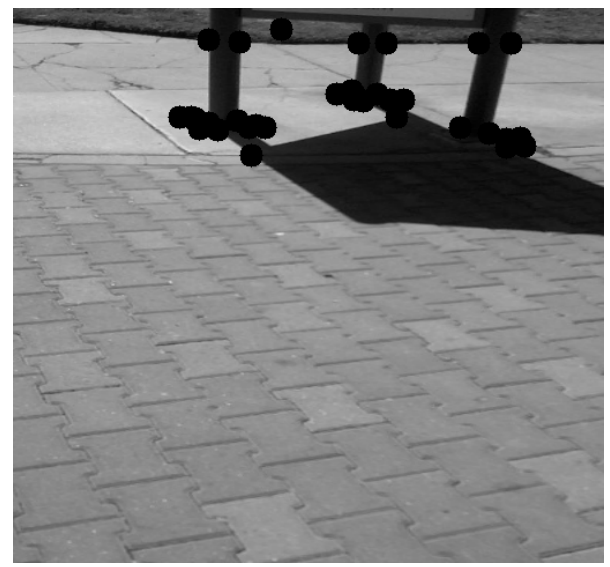

Figure 14: Feature point detection example for Waypoint tests

As for the Bike Locker tests, it had the best true postive rate of the three Single Object tests. The reason for this is the same as the reason why the Waypoint tests had a higher true positive rate than the Fire Hydranttests: it consistently found a large number of feature points clustered around the obstacle. Specifically, goodFeaturesToTrack would find collections of feature points clustered along the edge that segmented the ground from the bike lockers, as seen Figure 16.

\footnotetext{
${ }^{3}$ Detected feature points are denoted by black circles.
} 


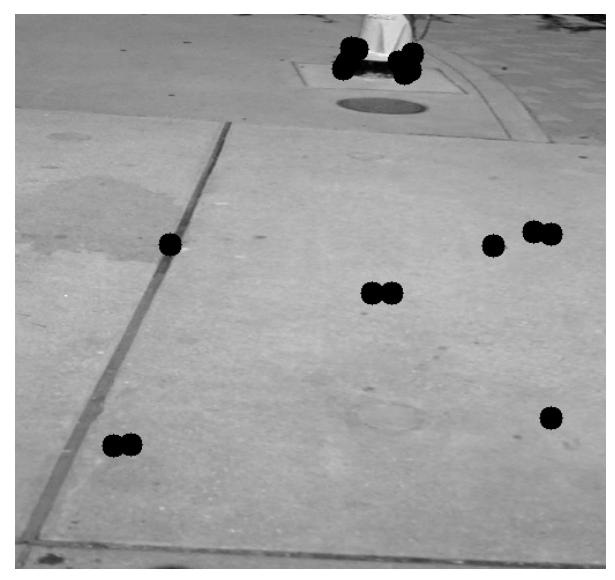

Figure 15: Feature point detection example for Fire Hydrant tests

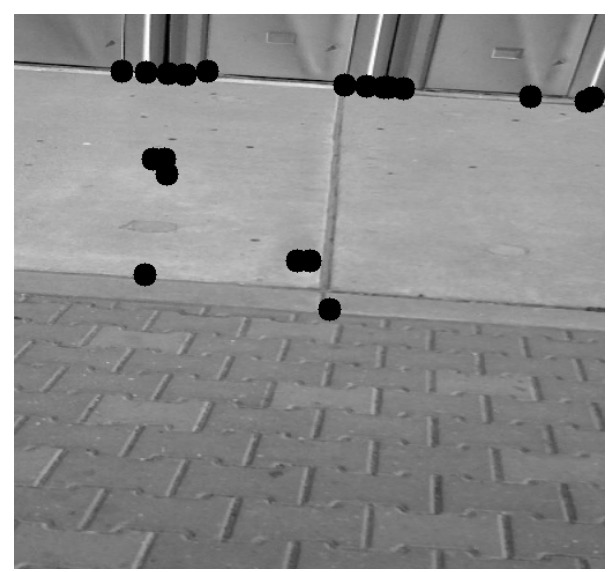

Figure 16: Feature point detection example for Bike Locker tests

\section{No Object Tests}

For each of the No Object tests, as there are no obstacles present during these tests, any user notifications given by the Walking Assistant are caused by false positive outcomes. For the Cobblestone tests, false positive identifications occurred $47.1 \%$ of the time, while the Sidewalk tests had a rate of $65.2 \%$ and the Asphalt tests had a rate of $35.3 \%$. The results for all of the Pre-Threshold No 


\begin{tabular}{|l|l|}
\hline Test \# & False Positive \\
\hline \hline 1 & $47.1 \%$ \\
\hline 2 & $65.2 \%$ \\
\hline 3 & $35.3 \%$ \\
\hline
\end{tabular}

Table 5: Results of the Pre-Threshold No Object tests

Object tests can be seen in Table $5^{4}$, where Test \#1 refers to the Cobblestone tests, Test \#2 refers to the Sidewalk tests, and Test \#3 refers to the Asphalt tests.

\section{Result Analysis}

The false positive identification rate of the Cobblestone tests is directly caused by the individual interlocking pieces that make up the cobblestone path. As the surface isn't made of large sections of concrete, the Walking Assistant finds a larger amount of feature points within the cobblestone path as the interlocking pieces of the path create a multitude of corners that goodFeatures To Track will pick up on, which can be seen in Figure 17. Plus, the corners tend to look so similar that optical flow may find corresponding feature points within the next frame even though, upon further review, they don't correspond to feature points found in the previous frame.

Unlike the Cobblestone tests, the false positive identification rate of Sidewalk tests isn't caused by the detection of feature points on the surface of the sidewalk, because, as previously mentioned, the average sidewalk is made up of large slabs of feature-less concrete. Thus, sidewalk typically lacks the corners that goodFeaturePointsToTrack would detect. Instead, the identification rate was caused by feature points found not on the sidewalk, either in the road on the left or in the dirt on the right. These areas were more likely to be detected as an obstacle due to the large amount of feature points clustered within those areas.

For the Asphalt tests, its false positive identification rate is largely due to

\footnotetext{
${ }^{4}$ As there are no obstacles present in this set of tests, the false positive rate is the only data produced, therefore the other fields are not displayed.
} 


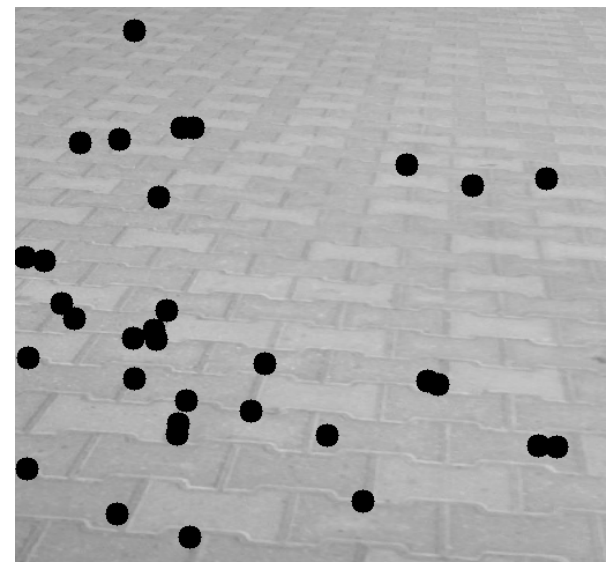

Figure 17: Feature point detection example for Cobblestone tests

different materials that make up an asphalt road. Specifically, 95\% of asphalt is made up of "aggregates," which includes "crushed stone, gravel, and sand" and the remaining $5 \%$ consists of asphalt cement, "the black liquid that acts as a glue to hold the pavement together" (Association 2014). Therefore, its inconsistent composition gives it some slight features, unlike sidewalk, which are detected.

\section{Shadow Tests}

Like the No Object tests, the Shadow tests lack obstacles for the Walking Assistant to detect, so any user notifications given by the Walking Assistant are caused by false positive identifications. For the Full Shadow tests, false positive notifications occurred $58.8 \%$ of the time, while the false positive rates of the Shadow Walk-Through and Scattered Shadows tests were $25.0 \%$ and $44.4 \%$, respectively. The results for all of the Pre-Threshold Shadow tests can be seen in Table $6^{5}$, where Test \#1 refers to the Full Shadow tests, Test \#2 refers to the Shadow Walk-Through tests, and Test \#3 refers to the Scattered Shadows tests.

\footnotetext{
${ }^{5}$ See Footnote 4.
} 


\begin{tabular}{|l|l|}
\hline Test \# & False Positive \\
\hline \hline 1 & $58.8 \%$ \\
\hline 2 & $25.0 \%$ \\
\hline 3 & $44.4 \%$ \\
\hline
\end{tabular}

\section{Table 6: Results of the Pre-Threshold Shadow tests}

\section{Result Analysis}

As the purpose of Full Shadow tests were to see if the presence of a constant shadow would affect the detection of feature points by goodFeatures To Track, the Walking Assistant performed well as it still picked up features in the surface the user was walking on - which were the cause of the false positive identifications despite the shadow.

For the Shadow Walk-Through tests, their purpose was similar to that of the Full Shadow tests, but it explored the effect of walking through a relatively solid shadow. For these tests, the surface of the ground was asphalt and thus, goodFeatures To Track produced a large amount of feature points, but the detection of feature points was affected by the shadow. As the user walks in and out of the shadow, the light intensities captured by the camera will vary, so regions of the ground appeared either lighter or darker than they actually were, as seen in Figure 18 . Therefore, goodFeaturesToTrack typically finds feature points only within one region, so in Figure 18a, feature points are only found in the lighter portion of the ground and not in the darker portion as it appears to be too dark to find any features. The opposite is true in Figure 18b; feature points aren't found within the lighter portion of ground as it appears too bright to find any features.

In the Scattered Shadows tests, a multitude of feature points were found on the sidewalk in addition to those found on the cracks in the sidewalk, as seen in Figure 19. As the shadows in these tests aren't nearly as solid as those in the previous two sets of tests, they had more of an effect on the amount of feature points that were detected. Since the boundary of a shadow transitions from a 


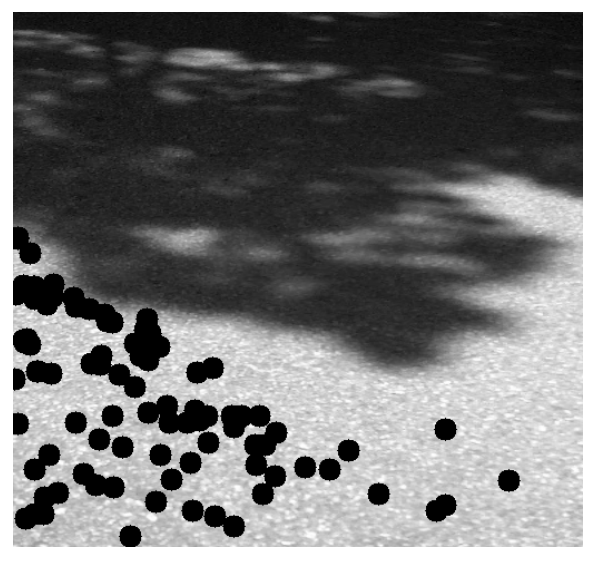

(a) Feature point detection while walking into a shadow

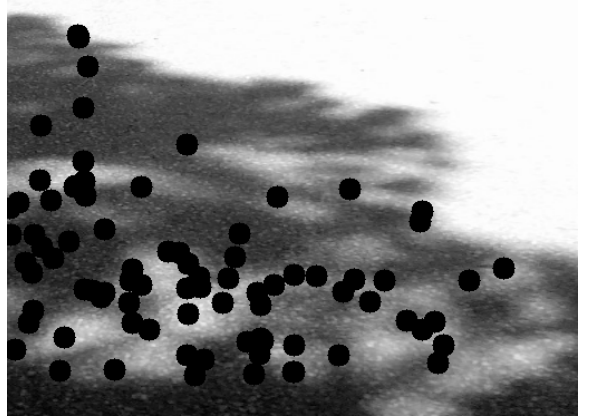

(b) Feature point detection while walking out of a shadow

Figure 18: Feature point detection example for Shadow Walk-Through tests

darker intensity to a lighter one, scattered shadows can create a dense patchwork of these transitions. Thus, when using the Shi-Tomasi corner detector through goodFeatures To Track, large differences can be found even if the search window has been shifted only slightly. As a result, the Shi-Tomasi corner detector will mark the region at the original location of the start window as a corner, so scattered shadows could potentially generate a large amount of feature points.

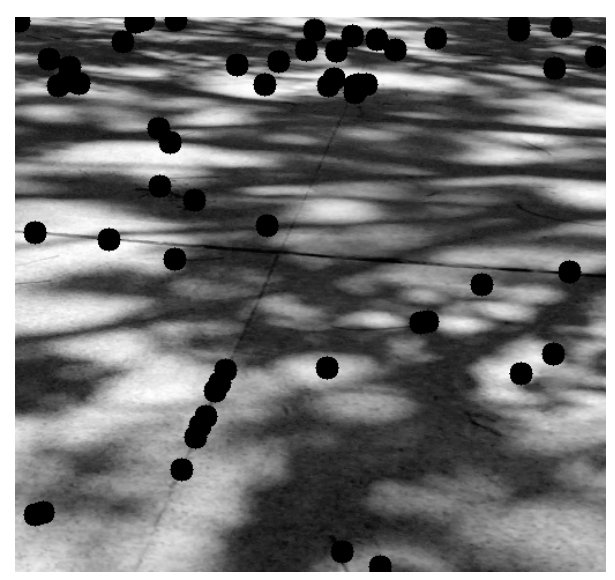

Figure 19: Feature point detection example for Scattered Shadows tests 


\begin{tabular}{|l|l|l|l|l|l|}
\hline Test \# & $\begin{array}{l}\text { Correct } \\
\text { ID }\end{array}$ & $\begin{array}{l}\text { Incorrect } \\
\text { ID }\end{array}$ & $\begin{array}{l}\text { True } \\
\text { Positive }\end{array}$ & $\begin{array}{l}\text { False } \\
\text { Positive }\end{array}$ & $\begin{array}{l}\text { False } \\
\text { Negative }\end{array}$ \\
\hline \hline 1 & $22.2 \%$ & $77.8 \%$ & $13.3 \%$ & $46.7 \%$ & $33.3 \%$ \\
\hline 2 & N/A & N/A & N/A & $52.6 \%$ & N/A \\
\hline
\end{tabular}

Table 7: Results of the Pre-Threshold Grass tests

\section{Grass Tests}

The Grass tests differ from the previous sets of tests as it only features two individual tests, one that features an obstacle - a fire hydrant - and one that doesn't feature any obstacles.

For the Grass Obstacle tests, $22.2 \%$ of the user notifications were cause by true positive outcomes, while $77.8 \%$ were caused by false positive outcomes. Additionally, the number of false positive outcomes $(46.7 \%)$ exceeded the amount of true positive (13.3\%) and false negative (33.3\%) outcomes. For the Grass Only tests, a higher false positive rate of $52.6 \%$ was produced. The results for all of the Pre-Threshold Grass tests can be seen in Table $7^{6}$, where Test \#1 refers to the Grass Obstacle tests and Test \#2 refers to the Grass Only tests.

\section{Result Analysis}

Regardless of the test, goodFeatures To Track consistently found a large amount of feature points, which could be attributed to the grass itself. As grass is made up of a multitude individual blades and each blade can cast a shadow and reflect the light of the sun, this could create an illusion of there being a large collection of corners on the grass due to the sudden changes from a darker intensity to a lighter one. This occurrence can be best seen in Figure 20, which displays an image captured during a Grass Only test. Due to the large amount of irrelevant feature points found on the grass, the Grass Obstacle tests had a low true positive rate and a high false positive rate. In the Grass Only tests, more false positive outcomes were generated as the only feature points that could be analyzed by

\footnotetext{
${ }^{6}$ See Footnote 4 for an explanation regarding the 'N/A' values for Test \#2.
} 
the collision warning process were those found on the grass, provided that optical flow found a large enough amount of corresponding feature points.

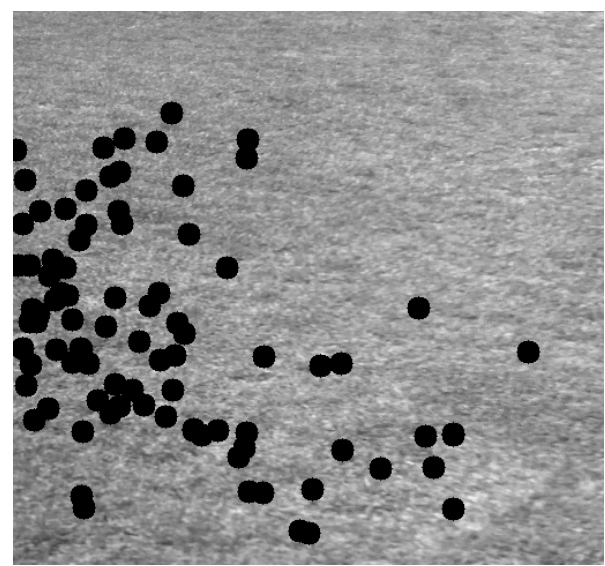

Figure 20: Feature point detection example for Grass Only tests

\section{Pre-Threshold Test Analysis}

After reviewing all of the data obtained during the Pre-Threshold tests, it was determined that for tests where an obstacle was present, the overall true positive rate was $46.7 \%$, while the overall false negative rate was $26.7 \%$ and the overall false positive rate was only $20.0 \%$. Additionally, the precision - the proportion of true positives against all of the positive results (true positives and false positives) - and sensitivity - the proportion of actual positives which are correctly identified as such - for these tests were $70.0 \%$ and $63.6 \%$, respectively. As for tests where an obstacle wasn't present, the overall false positive rate was $46.90 \%$. For a full view of the overall results of the Pre-Threshold tests, refer to Table $8^{7}$.

As thresholds for valid CP and TTC values weren't yet set during the PreThreshold tests, the false positive rates for the two overall types of tests could be potentially reduced once a set of thresholds are determined and implemented. Unfortunately, choosing a set of CP and TTC thresholds will mostly likely not

\footnotetext{
${ }^{7}$ As tests that lack an obstacle focus on reporting the false positive rates, the results of the "No Object Present" test type follows their example by reporting only the false positive rate.
} 


\begin{tabular}{|l|l|l|l|l|l|l|}
\hline $\begin{array}{l}\text { Test } \\
\text { Type }\end{array}$ & $\begin{array}{l}\text { True } \\
\text { Positive }\end{array}$ & $\begin{array}{l}\text { False } \\
\text { Positive }\end{array}$ & $\begin{array}{l}\text { True } \\
\text { Negative }\end{array}$ & $\begin{array}{l}\text { False } \\
\text { Negative }\end{array}$ & Precision & Sensitivity \\
\hline \hline $\begin{array}{l}\text { Object } \\
\text { Present }\end{array}$ & $46.7 \%$ & $20.0 \%$ & $6.6 \%$ & $26.7 \%$ & $70.0 \%$ & $63.6 \%$ \\
\hline $\begin{array}{l}\text { No } \\
\begin{array}{l}\text { Object } \\
\text { Present }\end{array}\end{array}$ & N/A & $46.9 \%$ & N/A & N/A & N/A & N/A \\
\hline
\end{tabular}

\section{Table 8: Overall results of the Pre-Threshold tests}

improve the true positive and false negative rates because the implementation of these thresholds will only further limit what is considered to be an obstacle. Therefore, the true positive rate may decrease, while the false negative rate increases, in the following Threshold tests.

After gathering together all of the CP, TTC pairs from each type of test, scatter plots were generated, displaying the ranges of the true positive and false positive CP, TTC pairs of each test, which can be seen in Figure 21. The majority of true positive CP, TTC pairs in the Single Object (Figure 21a) and Grass (Figure 21d) tests fall within a limited range, which could possibly define a target region of $\mathrm{CP}$ and TTC values that identify obstacles. On the other hand, the false positive CP, TTC pairs don't group within a specific range and occasionally fall within the range of true positive pairs. As for the false positive pairs in the No Object tests (Figure 21b), they have a much wider spread than their counterparts in the two aforementioned plots. The false positive CP, TTC pairs in the Shadow tests (Figure 21c) mimic their counterparts in the Single Object and Grass tests in that a collection of the pairs are grouped in the potential CP, TTC pair obstacle-identification region.

To obtain a better representation of the proposed target region, normal distributions for the true positive and false positive CP and TTC values were calculated and can be seen in Figures 22 and 23, respectively. Due to the wide range of false positive CP and TTC values, the normal distributions for these values don't necessarily create the ideal normal distribution. Therefore, to obtain a better view of the true positive CP and TTC distributions, the entire range of the false positive distributions isn't shown. As previously mentioned, there is a lack of a 


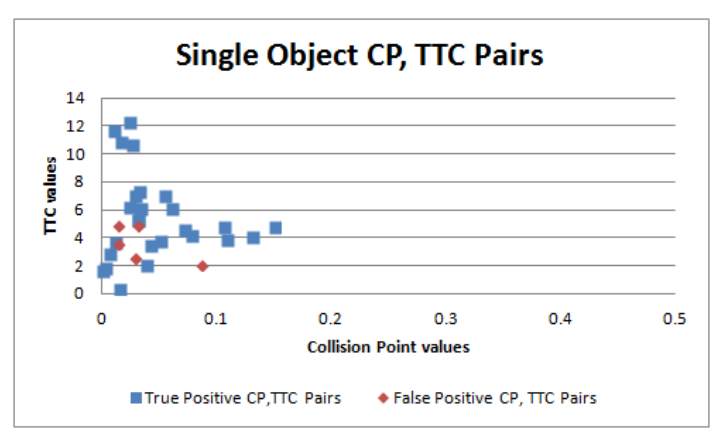

(a) Scatter plot of Pre-Threshold Single

Object CP, TTC pairs

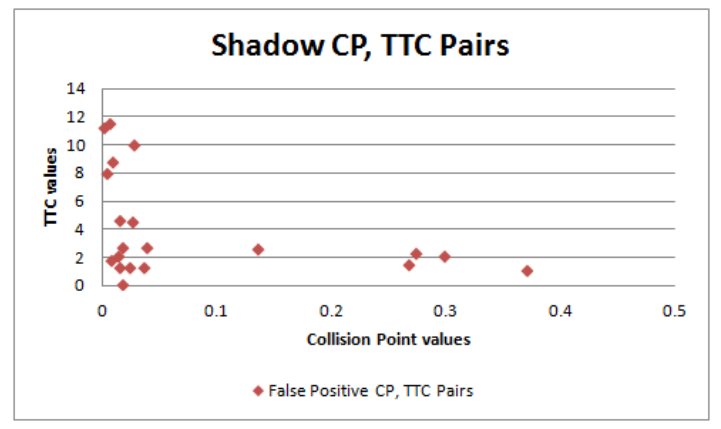

(c) Scatter plot of Pre-Threshold Shadow

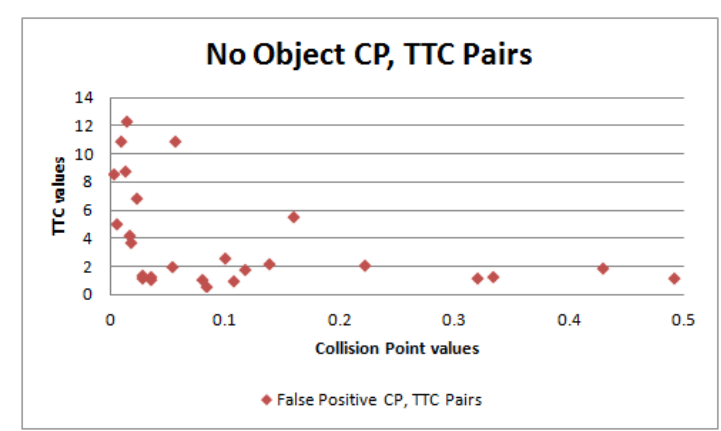

(b) Scatter plot of Pre-Threshold No Object $\mathrm{CP}$, TTC pairs

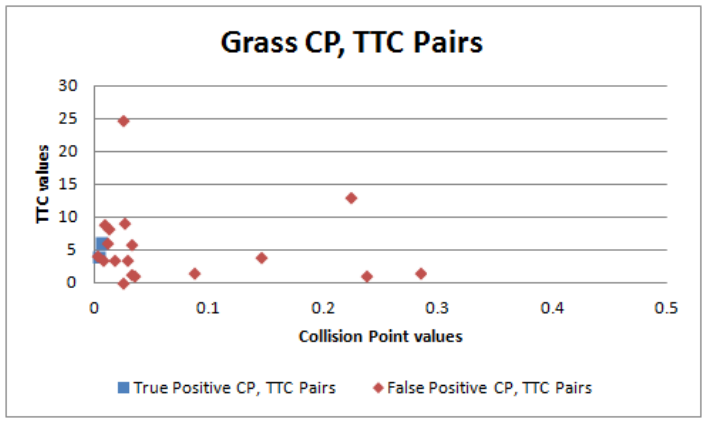

(d) Scatter plot of Pre-Threshold Grass CP, TTC pairs

Figure 21: Scatter plot of Pre-Threshold CP, TTC pairs by test type 
clear distinction as to what range of $\mathrm{CP}$ and TTC values refer to an oncoming obstacle, considering the overlap between true positive and false positive values.

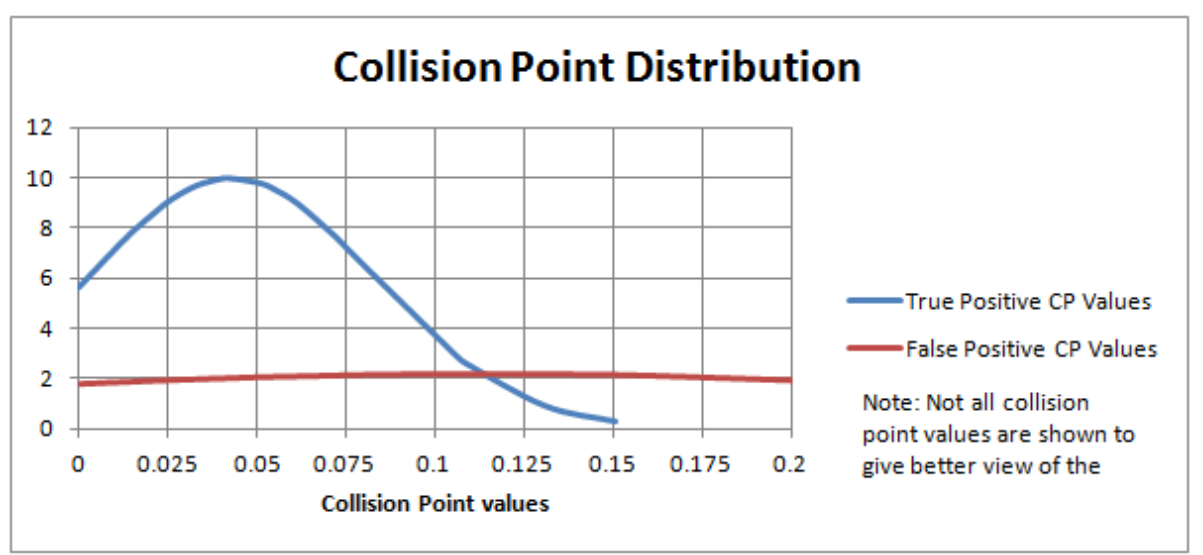

Figure 22: Distribution of true positive and false positive collision point values in the Pre-Threshold tests

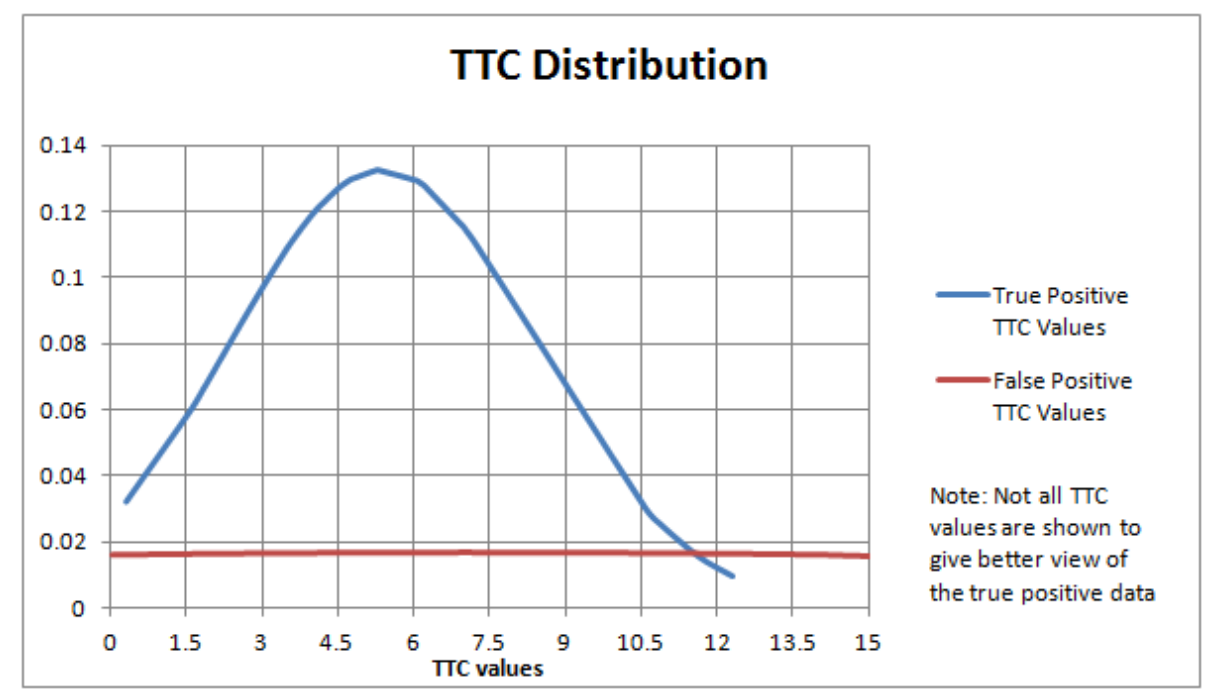

Figure 23: Distribution of true positive and false positive time-tocollision values in the Pre-Threshold tests

According to Figure 22, the only overlap between the two CP distributions occurs at the upper end of the true positive distribution. Therefore, the upper CP threshold will be set to the $\mathrm{CP}$ value at the intersection of the two distributions, 
which is approximately 0.11. As for the lower CP threshold, it is based upon the lower end of the true positive distribution, resulting in a value of 0 , but according to the data, this value is an outlier, so the next closest value of 0.003 is chosen.

As for the TTC distributions, its true positive and false positive distributions in Figure 23 are similar to those in Figure 22. Thus, the upper and lower TTC threshold values will be determined in the same manner. The upper TTC threshold is chosen to be 11.0 as it is the closest value to the distribution intersection. The lower threshold is initially chosen to be 0.3255 , but, as with the initial lower $\mathrm{CP}$ threshold, the value is an outlier, so the next closest value of 1.5 is chosen.

Now that the upper and lower thresholds for the CP and TTC values have been determined, the threshold set will be employed during the following Threshold tests, where 0.003 serves as lower CP threshold, 0.11 as the upper, 1.5 as the lower TTC threshold, and 11.0 as the upper.

\section{Threshold Test Results}

With the CP and TTC thresholds chosen, the same set of tests carried out for

the Pre-Threshold tests will be repeated, and the user will now be notified only if any of the feature points and its neighbors fall within the CP and TTC threshold ranges.

\section{Single Object Tests}

For the Fire Hydrant tests, the percentage of the user notification caused by true positive outcomes increased to $88.9 \%$ as a result of the number of true positives increasing by over $20 \%$ to $61.5 \%$. Additionally, the false positive rate dropped to $7.7 \%$, while the false negative rate increased to $30.8 \%$. For the Waypoint tests, its true positive rate increased slightly to $66.7 \%$, while its false negative rate remained unchanged at 33.3\%. The Bike Locker test outcome rates suffered the biggest changes as its true positive rate plummeted to $0 \%$, while its false positive and false negative rates increased sharply from $0 \%$ to $38.9 \%$ and $13.3 \%$ to $61.1 \%$, 


\begin{tabular}{|l|l|l|l|l|l|}
\hline Test \# & $\begin{array}{l}\text { Correct } \\
\text { ID }\end{array}$ & $\begin{array}{l}\text { Incorrect } \\
\text { ID }\end{array}$ & $\begin{array}{l}\text { True } \\
\text { Positive }\end{array}$ & $\begin{array}{l}\text { False } \\
\text { Positive }\end{array}$ & $\begin{array}{l}\text { False } \\
\text { Negative }\end{array}$ \\
\hline \hline 1 & $88.9 \%$ & $11.1 \%$ & $61.5 \%$ & $7.7 \%$ & $30.8 \%$ \\
\hline 2 & $100.0 \%$ & $0.0 \%$ & $66.7 \%$ & $0.0 \%$ & $33.3 \%$ \\
\hline 3 & $0.0 \%$ & $100.0 \%$ & $0.0 \%$ & $38.9 \%$ & $61.1 \%$ \\
\hline
\end{tabular}

Table 9: Results of the Threshold Single Object tests

respectively. The results for all of the Threshold Single Object tests can be seen in Table 9.

\section{Result Analysis}

As previously mentioned, it was expected that false positive rates would decrease while false negative rates would increase, but this trend was only observed in the results of the Fire Hydrant tests. As for the other two tests, the results of the Waypoint tests are nearly identical to those before CP and TTC thresholds were implemented, while the Bike Locker tests produced poor results in comparison to its pre-threshold counterpart.

The poor results of the Bike Locker tests can be attributed to the changing angle of the sun as its Pre-Threshold tests were carried out after 4 p.m. and its Threshold tests were carried out around 2:30 p.m. After comparing the recorded images from the Pre-Theshold and Threshold Bike Locker tests, it was discovered that during the Threshold tests, the side of the bike lockers facing the camera are in shadow, while the shadow wasn't present during the Pre-Threshold tests. Therefore, the presence of a shadow may have kept the bike lockers from being detected during the Threshold tests, possibly hiding the corners that were found during the Pre-Threshold tests.

\section{No Object Tests}

For the No Object tests, the false positive rates of each individual test decreased by at least $9 \%$. Of the three tests, the Cobblestone tests had the biggest change 


\begin{tabular}{|l|l|}
\hline Test \# & False Positive \\
\hline \hline 1 & $10.0 \%$ \\
\hline 2 & $32.0 \%$ \\
\hline 3 & $26.3 \%$ \\
\hline
\end{tabular}

Table 10: Results of the Threshold No Object tests

\begin{tabular}{|l|l|}
\hline Test \# & False Positive \\
\hline \hline 1 & $5.0 \%$ \\
\hline 2 & $14.2 \%$ \\
\hline 3 & $10.5 \%$ \\
\hline
\end{tabular}

Table 11: Results of the Threshold Shadow tests

in its false positive rate, dropping to $10.0 \%$, followed by the false positive rates of the Sidewalk and Asphalt tests, which were $32.0 \%$ and $26.3 \%$, respectively. The results of the Threshold No Object tests can be seen in Table $10^{8}$.

\section{Result Analysis}

The implementation of the CP and TTC thresholds had a clear effect on each of the No Object tests as the newly-implemented thresholds helped filter out $\mathrm{CP}$, TTC pairs that may have been previously recognized as an obstacle by the Walking Assistant.

\section{Shadow Tests}

Like the No Object tests before it, each of the Shadow tests produced lower false positive rates. The Full Shadow test had a false positive rate of $5.0 \%$, while the Shadow Walk-Through tests had a rate of $14.3 \%$ and the Scattered Shadow tests produced a rate of $10.5 \%$. The results for all of the Threshold Shadow tests can be seen in Table $11^{9}$.

\footnotetext{
${ }^{8}$ See Footnote 4

${ }^{9}$ See Footnote 4
} 


\begin{tabular}{|l|l|l|l|l|l|}
\hline Test \# & $\begin{array}{l}\text { Correct } \\
\text { ID }\end{array}$ & $\begin{array}{l}\text { Incorrect } \\
\text { ID }\end{array}$ & $\begin{array}{l}\text { True } \\
\text { Positive }\end{array}$ & $\begin{array}{l}\text { False } \\
\text { Positive }\end{array}$ & $\begin{array}{l}\text { False } \\
\text { Negative }\end{array}$ \\
\hline \hline 1 & $75.0 \%$ & $25.0 \%$ & $40.0 \%$ & $13.3 \%$ & $26.7 \%$ \\
\hline 2 & N/A & N/A & N/A & $31.3 \%$ & N/A \\
\hline
\end{tabular}

Table 12: Results of the Threshold Grass tests

\section{Result Analysis}

Much like the No Object tests, the false positive rates of the Shadow tests underwent a change once the CP and TTC thresholds were implemented and its effects were quite dramatic.

\section{Grass Tests}

When compared to its Pre-Threshold tests, the Grass Obstacle and Grass Only tests each improved in different ways. As its true positive rate increased to $40.0 \%$ and its false positive rate decreased to $13.30 \%$, the Grass Obstacle tests featured a rise how often it notified the user about a correctly-identified obstacle, from $22.2 \%$ to $75.0 \%$. As for the Grass Only tests, its false positive rate dropped from $52.6 \%$ to $31.3 \%$. The full results for the Threshold Grass tests can be seen in Table $12^{10}$.

\section{Result Analysis}

While it may initially appear that the improved results of the Grass tests are due to the implemented CP and TTC thresholds, the results may have been affected by the time of day when the tests were carried out. The Pre-Threshold Grass tests were carried out around 3 p.m., while the Threshold tests were carried out closer to 1 p.m. Therefore, the shadows cast by the blades of grass during may have been less prominent during the Threshold tests when the sun was overhead. As a result, the smaller amount of "grass" feature points - in comparison to the

\footnotetext{
${ }^{10}$ See Footnote 4 for an explanation regarding the 'N/A' values for Test \#2.
} 


\begin{tabular}{|l|l|l|l|l|l|l|}
\hline $\begin{array}{l}\text { Test } \\
\text { Type }\end{array}$ & $\begin{array}{l}\text { True } \\
\text { Positive }\end{array}$ & $\begin{array}{l}\text { False } \\
\text { Positive }\end{array}$ & $\begin{array}{l}\text { True } \\
\text { Negative }\end{array}$ & $\begin{array}{l}\text { False } \\
\text { Negative }\end{array}$ & Precision & Sensitivity \\
\hline \hline $\begin{array}{l}\text { Object } \\
\text { Present }\end{array}$ & $42.1 \%$ & $15.0 \%$ & $12.5 \%$ & $30.4 \%$ & $70.6 \%$ & $50.0 \%$ \\
\hline $\begin{array}{l}\text { No } \\
\begin{array}{l}\text { Object } \\
\text { Present }\end{array}\end{array}$ & N/A & $18.5 \%$ & N/A & N/A & N/A & N/A \\
\hline
\end{tabular}

Table 13: Overall results of the Threshold tests

large amounts found during the Pre-Threshold tests - may have had a lesser effect on skewing the results as feature points grouped in dense clusters had higher CP and TTC values based on their neighborhood. This, in turn, might have allowed the CP and TTC values of the feature points that referred to the fire hydrant in the Grass Object test to stand out.

\section{Threshold Test Analysis}

Following the execution of all of the Threshold tests, the four outcome rates for when an obstacle was and wasn't present were calculated and are listed in Table $13^{11}$. It was calculated that, for tests where an obstacle was present, the overall true positive rate decreased to $42.1 \%$, the false positive rate decreased to $15.0 \%$, and the false negative rate increased to $30.4 \%$. For tests when an obstacle wasn't present, it was found that the false positive rate fell to $18.5 \%$. As for the precision and sensitivity ratios, precision increased to $70.6 \%$, while sensitivity dropped to $50.0 \%$.

The overall outcome rates fall in line with the expectations described in Section VII., where, after implementing the CP and TTC thresholds, the false positive and true negative rates would improve - which they did by, respectively, decreasing by $5 \%$ when an obstacle was present and decreasing by $28.4 \%$ when an obstacle wasn't present, and increasing by $5.9 \%$. The improvement of the false positive rate is also seen in the precision ratio as it increased slightly by $0.6 \%$. It was also expected that the false negative and true positive rates when an object

\footnotetext{
${ }^{11}$ See Footnote 7
} 
was present would become worse, which was supported as the true positive rate was reduced by $4.6 \%$ and the false negative rate rose by $3.7 \%$. The worsening of these two rates are also seen in the sensitivity ratio as it decreased - more so than the precision ratio increased - by $13.6 \%$.

Besides being affected by the newly-implemented thresholds, the changes in outcome rates may have also been affected by the time of day when the PreThrehsold and Threshold tests were carried out and, therefore, the angle of the sun. As the angle of the sun can cause shadows to grow or recede, these changing shadows can either hide object features - such as the edges of the bike lockers as described in Section VII. - or create extra noise within an image in the form of additional feature points - like those caused by scattered shadows or the shadows of blades of grass as described, respectively, in Sections VII. and VII..

It's important that the false positive rate is kept to a minimum for when a user is notified, it serves as a cue to be aware of an upcoming object. Thus, a high rate of false positives can cause the user to be constantly alerted and hindering their journey to their destination. Plus, the low true positive rate of $42.1 \%$ means that it simply doesn't always identify the obstacle in every frame it is present in. Considering the Threshold Fire Hydrant and Waypoint tests, the obstacle was detected at least twice in all of the tests. Therefore, the user is still being notified about the obstacle, but the obstacle isn't constantly being detected in every image captured, which is the primary cause of the low true positive rate. 


\section{Conclusion}

Overall, the Walking Assistant is successful in that it can detect obstacles with a degree of accuracy and can notify the user about said obstacle if the app determines the obstacle will collide with the user. Thus, the app can help give visually-impaired users peace of mind as they walk. However, the accuracy of the Walking Assistant is limited by difficulties associated with outdoor environments, limitations of the smartphone's hardware, and limitations of optical flow. The app can greatly benefit from some additional work that can improve both its accuracy and usability.

In order for the user to recieve the biggest benefit from the app, it is recommended that the Walking Assistant be run on a wide and relatively feature-less surface such as sidewalk - thus, not on asphalt or grass - with, if possible, no shadows covering it. Additionally, to keep the user's shadow from interfering with the app's operation, the sun should be either overhead or in front of the user.

\section{Difficulties and Limitations}

As the Walking Assistant is based in the field of computer vision, a number of potential difficulties can arise depending on which aspect of computer vision is focused on. In this case, the Walking Assistant deals with gathering and analyzing information in an image taken of an outdoor environment. 


\section{Outdoor Environment Difficulties}

The Walking Assistant operates by gathering and analyzing information in an image taken of an outdoor environment, which is much more difficult than analyzing an indoor environment. Difficulties arise due to the number and range of attributes present in outdoor environments and the challenges involved in controlling them. In an indoor setting, one has to deal with significantly less environmental attributes and can more easily control them. For example, one doesn't have to deal with the changing angle of the sun while indoors and can easily adjust the amount of light within a room with the flick of a switch.

For the Walking Assistant, the biggest difficulties presented by an outdoor environment include the variance in illumination and the type of surface the user is walking on. While outdoors, the amount of illumination is determined by the weather and essentially unconstrained, as there's more light available on a sunny day as opposed to a day with overcast skies. Additionally, the amount of light in the environment can also be altered by the angle of the sun, causing the outdoor environment to be lit either by direct or indirect sunlight.

The sun's angle can also create shadows, which can skew results of the object detection process. Shadows can either hide objects, like in Figure 24, that would have normally been detected or create "false" objects, like in Figure 19.

Objects can be hidden if the locations of feature points have been covered in shadows from one image to the next, thereby altering the pixel intensity at those locations. As a result, feature points found in one image are not found in the next as optical flow finds feature point locations in following images by looking for regions with pixel intensities that match those found in the first image. Shadows can create "false" objects in that objects are found by searching an image for well-defined corners, which shadows can inadvertently create. Thus, a shadow on the ground may appear as an object to the Walking Assistant app, which will cause the app to alert the user despite the absence of an object.

Lastly, the type of surface the user is walking on while the Walking Assistant is running can also skew the object detection results. As detailed in Sections VII. 


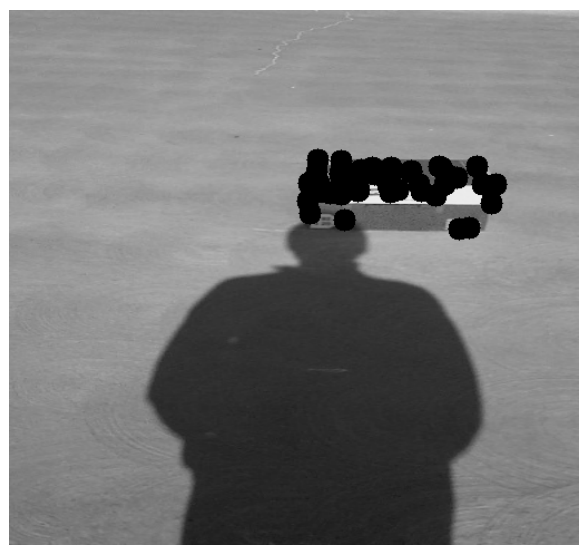

(a) Before an object is hidden by a shadow cast by the user

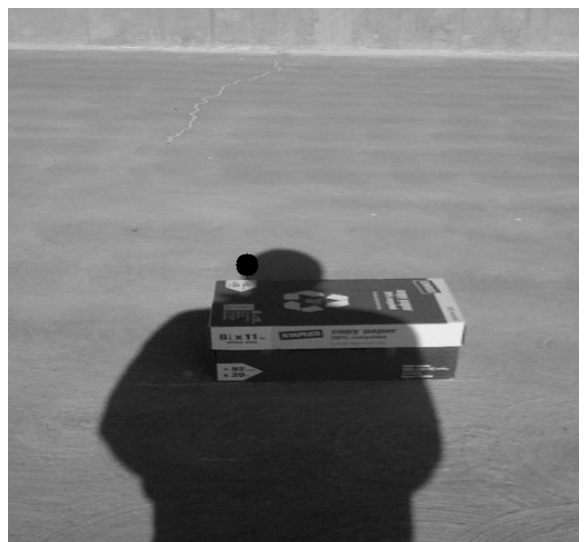

(b) After an object is hidden by a shadow cast by the user

\section{Figure 24: An example of how a shadow can hide a potential obstacle}

and VII., some surface types can create noise due to surface's composition. For example, on a cobblestone path, as seen in Figure 17, the Walking Assistant may determine the cracks between each stone to be a corner, creating an abundance of extra feature points that the app must then analyze. This effect is more prevalent if the user is walking on grass, as seen in Figure 20, as the blades of grass each cast their own shadow, which, as mentioned earlier, will be considered a corner and, thus, potentially part of an object.

In essence, the Walking Assistant lacks context; it doesn't know the angle of the sun or what type of surface the user is walking on. If the Walking Assistant had some context about the environment the user was walking through, its operation could be better tuned, increasing the accuracy of the object detection process. This additional context could be obtained by either limiting the scope of the app to a context or making use of extra, more sophisticated sensors to get more information about the environment. Limiting the scope of the app though would mean reducing its applicability, while using more sensors depends on what is available on the user's smartphone - which is discussed further in the following section. 


\section{Hardware Limitations}

The Walking Assistant is also limited by the smartphone hardware it is being run on. As testing was conducted using a single smartphone - the Droid 4 - and a wide variety of Android-based smartphones are available, it wasn't deteremined exactly which smartphones could execute the Walking Assistant. Yet, with smartphone hardware becoming more and more powerful, newly-released smartphones should be more than capable.

Another limitation that must be considered is how much energy the Walking Assistant consumes. Due to the Walking Assistant's continuous use of the smartphone's camera and its computation-heavy nature, the app could quickly drain a smartphone's battery power. At the same time, the app's energy consumption is reduced as it doesn't use the smartphone's screen. Thus, the screen never needs to be updated and can be set to the lowest screen brightness setting without affecting a user's interaction with the app.

A standard smartphone also limits the full potential of the Walking Assistant as it lacks the sophisticated sensors used in other computer vision-based navigation systems, such as the Google's driverless car. According to (Guizzo 2011), Google's driverless car can accurately drive itself through the use of following sensors: a Velodyne 64-beam laser ${ }^{1}$, four radars, a camera, a GPS, an inertial measurement unit, and a wheel encoder. Of the six types of sensors Google's driverless car uses, a smartphone typically only has two, a camera and a GPS, but some have accelerometers, gyroscopes, and light sensors, which senses how much light is within its immediate surroundings. The lack of sophisticated sensors on a smartphone limits the amount of information it can gather from an environment, thereby hindering its ability to accurately detect objects in the user's path.

\footnotetext{
${ }^{1}$ Google's driverless car uses a Velodyne 64 -beam laser to generate a detailed 3D map of the environment.
} 


\section{Optical Flow Limitations}

Optical flow plays a key role within the Walking Assistant, as the app revolves around determining if an object is moving towards the camera, so the limitations of optical flow can drastically affect the app's object detection accuracy. In order to carry out its calculations, the optical flow algorithm must make a few assumptions, one of which is: "the pixel intensities of an object do not change between consecutive frames" (Team c). If this assumption isn't met, an object, whose pixel intensities have changed, could essentially appear to have disappeared from view. For example, in the context of the Walking Assistant, if a box in the user's path is suddenly covered by a shadow, the app will no longer consider the box to be a potential obstacle as its pixel color values have been darkened by the shadow.

Additionally, optical flow operates by attempting to "minimize the difference" between regions in the first image and regions in the second image (Baker and Matthews 2004). Thus, the corresponding feature points found in the second image are estimations - hence the returned error values in calcOpticalFlowPyrLK -, all of which can be wildly inaccurate. A threshold is used to filter out potentially inaccurate feature points, but it is far from a catch-all, so some inaccurate points may slip through and skew the results of the object detection process.

\section{Future Work}

To further increase the robustness of the Walking Assistant app, a number of features could be implemented. First, a person's acceleration could be measured using a smartphone's accelerometer and used to dynamically update the TTC threshold accordingly. Alternatively, both the collision point and TTC threshold could be updated using a fuzzy decision system presented in Pundlik and Luo's object detection process. Second, as the user will have their smartphone mounted on their body through the Go Pro Chest Harness, image stabilization could be applied to correct for the bouncing of the camera, using a gyroscopic sensor, like 
in Pundlik et al.'s object detection process, or a fuzzy-based stabilization system described in the work of Yi-Ying Shih et al. (Shih, Su, and Rudas 2012). Third, a sidewalk mode could be added, which would be specifically tailored to detect objects on sidewalks. This mode would make use of John Seng and Thomas Norrie's sidewalk identification algorithm and could possibly speed up the object detection process by focusing on detecting objects on the sidewalk rather than the entire image(Seng and Norrie 2008).

Besides adding more features to the Walking Assistant, extra testing could also be carried out to improve its usability and measure its energy consumption. In regards to app usability, conducting usability evaluations with visuallyimpaired people could provide some valuable insight as to how the average user might use the app and if any alterations are required to improve ease of interaction. A brief outline of a proposed usability evaluation for the Walking Assistant can be found in Appendix A. Gathering energy consumption measurements for the Walking Assistant would also prove beneficial as the app could potentially drain a smartphone's battery. The app's energy consumption is worth investigating as the app is intended to be run for longer periods of time than the average app and can be computationally intensive, but doesn't utilize the smart-

phone's screen. Rajesh Palit et al's and A. Abogharaf et al.'s work both present a viable methodology for measuring an app's energy consumption, where energy consumption is measured in combination with various energy-consuming smartphone settings (Palit, Arya, Naik, and Singh 2011; Abogharaf, Palit, Naik, and Singh 2012).

\section{Closing Remarks}

The proposed Walking Assistant app is an easy-to-use, accessible alternative navigation aid since it doesn't require any special or overly expensive equipment besides an Android smartphone, a harness to hold the smartphone, and the OpenCV library. The results of the suite of tests carried out The Walking Assistant can also operate within a real-world environment with a fair amount of 
accuracy, despite the difficulties of operating in a real-world context and the limitations of the hardware and algorithms it uses. Its accuracy and usability can be further refined through additional work, which could include implementing fuzzy thresholding or holding usability evaluations. 


\section{Bibliography}

jdt - java delaunay triangulation.

Abogharaf, A., R. Palit, K. Naik, and A. Singh (2012, June). A methodology for energy performance testing of smartphone applications. In Automation of Software Test (AST), 2012 7th International Workshop on, pp. 110-116.

Amazon (2014). Amazon.com: iglasses ultrasonic mobility aid- tinted lens: Health \& personal care.

AmbuTech (2014). Custom mobility canes and aids for the blind and visually impaired.

Association, N. A. P. (2014). An asphalt plant in your community?

Baker, S. and I. Matthews (2004). Lucas-kanade 20 years on: A unifying framework. International Journal of Computer Vision 56(3), 221-255.

Corporation, O. (2014). Synchronization (the java tutorials - essential classes - concurrency).

Culjak, I., D. Abram, T. Pribanic, H. Dzapo, and M. Cifrek (2012, May). A brief introduction to opencv. In MIPRO, 2012 Proceedings of the 35th International Convention, pp. 1725-1730.

Derpanis, K. G. (2005). The gaussian pyramid.

Eklundh, J.-O. and H. Christensen (2001). Computer vision: Past and future. In R. Wilhelm (Ed.), Informatics, Volume 2000 of Lecture Notes in Computer Science, pp. 328-340. Springer Berlin Heidelberg.

Ersi. Arcgis help 10.1 - what is a tin surface? 
Fortune, S. (1997). Handbook of discrete and computational geometry. Chapter Voronoi Diagrams and Delaunay Triangulations, pp. 377-388. Boca Raton, FL, USA: CRC Press, Inc.

Gjacquenot (2013). Delaunay circumcircles vectorial.

Guizzo, E. (2011, Oct). How google's self-driving car works. http://spectrum.ieee.org/automaton/robotics/artificial-intelligence/howgoogle-self-driving-car-works.

Hanlon, M. (2013a, November). The evolution of nsk's guide robot for the visually-impaired.

Hanlon, M. (2013b, November). The evolution of nsk's guide robot for the visually-impaired.

Harris, C. and M. Stephens (1988). A combined corner and edge detector. In In Proc. of Fourth Alvey Vision Conference, pp. 147-151.

Heggestuen, J. (2013, December). Smartphone and tablet penetration.

Itseez (2014). About - opencv. http://opencv.org/about.html.

Jie, Y. and S. Yanbin (2012, Aug). Obstacle detection of a novel travel aid for visual impaired people. In Intelligent Human-Machine Systems and Cybernetics (IHMSC), 2012 4th International Conference on, Volume 2, pp. $362-364$.

Liyanage, D. K. and M. U. S. Perera (2012, April). Optical flow based obstacle avoidance for the visually impaired. In Business Engineering and Industrial Applications Colloquium (BEIAC), 2012 IEEE, pp. 284-289.

Ltd, S. F. T. About the ultracane.

Ltd, S. F. T. (2012, January). Ultracane user guide v1.6.

Lucas, B. D. and T. Kanade (1981). An iterative image registration technique with an application to stereo vision. In In IJCAI81, pp. 674-679.

McGirr, R. Sonar traveller cane - sonar cane for the blind.

Ni, D., L. Wang, Y. Ding, J. Zhang, A. Song, and J. Wu (2013, Dec). The design and implementation of a walking assistant system with vibrotac- 
tile indication and voice prompt for the visually impaired. In Robotics and Biomimetics (ROBIO), 2013 IEEE International Conference on, pp. 27212726.

of Education, C. D. (2010). Aph product detail - imods (ca department of education).

Oudot, S. Delaunay triangulation.

Palit, R., R. Arya, K. Naik, and A. Singh (2011). Selection and execution of user level test cases for energy cost evaluation of smartphones. In Proceedings of the 6th International Workshop on Automation of Software Test, AST '11, New York, NY, USA, pp. 84-90. ACM.

Pundlik, S. and G. Luo (2012, May). Collision risk estimation from an uncalibrated moving camera based on feature points tracking and clustering. In In Proc. 2012 9th International Conference on Fuzzy Systems and Knowledge Discovery, pp. 550-554.

Seng, J. S. and T. J. Norrie (2008, June). Sidewalk following using color histograms. J. Comput. Sci. Coll. 23(6), 172-180.

Shi, J. and C. Tomasi (1994). Good features to track.

Shih, Y.-Y., S.-F. Su, and I. Rudas (2012, June). Fuzzy based hand-shake compensation for image stabilization. In System Science and Engineering (ICSSE), 2012 International Conference on, pp. 40-44.

Shrinivas Pundlik, M. T. and G. Luo (2013, June). Collision detection for visually impaired from a body-mounted camera. In In Proc. of 2013 IEEE Conference on Computer Vision and Pattern Recognition Workshops, pp. $41-47$.

Song, K.-T. and J.-H. Huang (2001). Fast optical flow estimation and its application to real-time obstacle avoidance. In Robotics and Automation, 2001. Proceedings 2001 ICRA. IEEE International Conference on, Volume 3, pp. 2891-2896 vol.3.

Team, O. D. Feature detection - opencv 2.4.9.0 documentation. 
Team, O. D. Motion analysis and object tracking - opencv 2.4.9.0-dev documentation.

Team, O. D. Optical flow - opencv 3.0.0-dev documentation.

Team, O. D. Shi-tomasi corner detector \& good features to track - opencv 3.0.0-dev documentation.

Zabonne. Zabonne - k sonar. 
Appendices 


\section{A Walking Assistant Usability Evaluation Outline}

\section{Evaluation Goals and Objectives}

The goal of the Walking Assistant is to better assist visually-impaired users in navigating through an outdoor environment, so the app must be beneficial to the user, rather than a burden, and must be easy to learn and control. The usability evaluation is carried out to determine if the Walking Assistant app meets these goals and, if not, how the usability of the app can be improved.

\section{Methodology}

Since the app revolves around assisting its users, which happens to be a unique group of users, holding usability experiments with the targeted users as participants is of the utmost importance. As such, visually-impaired users, who make use of white canes to navigate outdoors, will be sought out. The usability tests will involve having the users use The Walking Assistant in conjunction with their white cane to navigate outdoors, and collecting feedback and recommendations based on their experience with my app.

\section{Usability Evaluation Plan}

For the participants, a potential source of volunteers could be the visuallyimpaired members of the Central Coast Assistive Technology Center, but if no volunteers are found there, blindfolded volunteers can be used as substitutes. 
For the evaluation environment, the evaluations will be run on section of sidewalk and, for best results, during a bright, sunny day.

For the evaluation itself, three different types of evaluations will be carried out: one for the vocal command aspect of the app, another for the touch command aspect, and one for the object detection and collision warning aspect. For the vocal command aspect, the experimenter will describe how to start the voice recognition in the app and the valid voice commands the user can give, and then have the user try out the voice commands for themselves. For the touch command aspect, the test will follow the same format as the previous vocal command tests, but the user will instead use touch-based commands. For the object detection and collision warning aspect, the experimenter will have the user walk back and forth across the section of sidewalk, with each traversal serving as a different test. These tests could include placing object directly in front of the user, to either side of the user, or have no obstacles altogether. Control experiments will also be held where the user doesn't use the app at all and uses just their white cane to navigate.

Following both of these evaluations, the experimenter will ask a series of follow-up questions regarding ease of use and effectiveness, in addition to collecting any general comments about the Walking Assistant. 